\title{
Vacuum Expectation Values of the Quantum Fields
}

\author{
Yury M. Zinoviev*
}

Steklov Mathematical Institute, Gubkin St. 8, 119991, Moscow, Russia, e - mail: zinoviev@mi.ras.ru

\begin{abstract}
The new axiomatic system for the quantum field theory is proposed. The new axioms are the description of the distributions. For the finite series these distributions satisfy the linear Wightman axioms.
\end{abstract}

\section{Introduction}

Gårding - Wightman axioms [1] are the mathematical form of the physical views of the quantum fields. Let us describe the finite dimensional irreducible representations of the group $S L(2, \mathbf{C})$, consisting of the complex $2 \times 2$ - matrices

$$
A=\left(\begin{array}{cc}
A_{11} & A_{12} \\
A_{21} & A_{22}
\end{array}\right)
$$

with the determinant equal to 1 . The complex conjugate of the matrix (1.1) is

$$
\bar{A}=\left(\begin{array}{cc}
\bar{A}_{11} & \bar{A}_{12} \\
\bar{A}_{21} & \bar{A}_{22}
\end{array}\right) \text {. }
$$

Let us consider the values $\xi_{\alpha_{1} \cdots \alpha_{j} \dot{\beta}_{1} \cdots \dot{\beta}_{k}}$ with the indices $\alpha$ and $\dot{\beta}$ equal to 1,2 . The value $\xi$ is symmetric under the permutations of the indices $\alpha$ and the permutations of the indices $\dot{\beta}$. For any matrix (1.1) we define the linear transformation

$$
\sum_{(\rho)(\dot{\sigma})}\left(\prod_{s=1}^{j} A_{\alpha_{s} \rho_{s}}\right)\left(\prod_{t=1}^{k} \bar{A}_{\dot{\beta}_{t} \dot{\sigma}_{t}}\right) \xi_{\rho_{1} \cdots \rho_{j} \dot{\sigma}_{1} \cdots \dot{\sigma}_{k}} .
$$

The dot over the index means simply that the value with this index transforms with a matrix $\bar{A}$; the symbol $(\rho)$ means $\rho_{1} \cdots \rho_{j}$; the symbol $(\dot{\sigma})$ means $\dot{\sigma}_{1} \cdots \dot{\sigma}_{k}$. This representation

*This work was supported in part by the Russian Foundation for Basic Research (Grant No. 07 - 01 00144) and Scientific Schools 672.2006.1 
of the group $S L(2, \mathbf{C})$ is denoted by $D^{(j / 2, k / 2)}(A, \bar{A})$. Any finite dimensional irreducible representation of the group $S L(2, \mathbf{C})$ is equivalent to one of these representations.

Due to the book [2] we formulate the properties of the vacuum expectation values of the products of the quantum fields.

\section{Spectrality}

The vacuum expectation value of the product of $n+1, n=1,2, \ldots$, quantum field is the distribution

$$
\begin{array}{r}
W_{(\alpha)(\dot{\beta}), \ldots,(\gamma)(\dot{\delta})}\left(x_{2}-x_{1}, \ldots, x_{n+1}-x_{n}\right)= \\
\int d^{4 n} p \widetilde{W}_{(\alpha)(\dot{\beta}), \ldots,(\gamma)(\dot{\delta})}\left(p_{1}, \ldots, p_{n}\right) \exp \left\{i \sum_{j=1}^{n}\left(p_{j}, x_{j+1}-x_{j}\right)\right\} \\
(x, y)=x^{0} y^{0}-\sum_{k=1}^{3} x^{k} y^{k} .
\end{array}
$$

The distribution

$$
\widetilde{W}_{(\alpha)(\dot{\beta}), \ldots,(\gamma)(\dot{\delta})}\left(p_{1}, \ldots, p_{n}\right) \in S^{\prime}\left(\mathbf{R}^{4 n}\right)
$$

has the support in the product of the closed upper light cones

$$
\bar{V}_{+}=\left\{x \in \mathbf{R}^{4}: x^{0} \geq 0,(x, x) \geq 0\right\} .
$$

The matrix

$$
A^{T}=\left(\begin{array}{ll}
A_{11} & A_{21} \\
A_{12} & A_{22}
\end{array}\right)
$$

is called the transposed matrix. The matrix $A^{*}=(\bar{A})^{T}$ is called Hermitian adjoint matrix. If $A^{*}=A$, the matrix (1.1) is Hermitian. Let us consider the basis of the Hermitian $2 \times 2$ matrices

$$
\sigma^{0}=\left(\begin{array}{ll}
1 & 0 \\
0 & 1
\end{array}\right), \sigma^{1}=\left(\begin{array}{ll}
0 & 1 \\
1 & 0
\end{array}\right), \sigma^{2}=\left(\begin{array}{cc}
0 & -i \\
i & 0
\end{array}\right), \sigma^{3}=\left(\begin{array}{cc}
1 & 0 \\
0 & -1
\end{array}\right)
$$

We relate with a vector $x \in \mathbf{R}^{4}$ an Hermitian matrix

$$
\tilde{x}=\sum_{\mu=0}^{3} x^{\mu} \sigma^{\mu} .
$$

We identify a vector $x \in \mathbf{R}^{4}$ and an Hermitian matrix (1.10).

\section{Lorentz covariance}

For any matrix (1.1)

$$
\begin{array}{r}
\widetilde{W}_{(\alpha)(\dot{\beta}), \ldots,(\gamma)(\dot{\delta})}\left(A \tilde{p}_{1} A^{*}, \ldots, A \tilde{p}_{n} A^{*}\right)= \\
\sum_{\left(\alpha^{\prime}\right)\left(\dot{\beta}^{\prime}\right), \ldots,\left(\gamma^{\prime}\right)\left(\dot{\delta}^{\prime}\right)} D_{(\alpha)(\dot{\beta}),\left(\alpha^{\prime}\right)\left(\dot{\beta}^{\prime}\right)}^{\left(j_{1} / 2, k_{1} / 2\right)}(A, \bar{A}) \cdots D_{(\gamma)(\dot{\delta}),\left(\gamma^{\prime}\right)\left(\dot{\delta}^{\prime}\right)}^{\left(j_{n+1} / 2\right)}(A, \bar{A}) \widetilde{W}_{\left(\alpha^{\prime}\right)\left(\dot{\beta}^{\prime}\right), \ldots,\left(\gamma^{\prime}\right)\left(\dot{\delta}^{\prime}\right)}\left(\tilde{p}_{1}, \ldots, \tilde{p}_{n}\right) .
\end{array}
$$

\section{Locality}

Let $\pi$ be a permutation of the natural numbers $1, \ldots, n+1$. If $\left(x_{i}-x_{j}, x_{i}-x_{j}\right)<0$ for all numbers $i, j$ whose order is changed by a permutation $\pi$, then

$$
\begin{array}{r}
W_{\pi ;(\alpha)(\dot{\beta}), \ldots,(\gamma)(\dot{\delta})}\left(x_{\pi(2)}-x_{\pi(1)}, \ldots, x_{\pi(n+1)}-x_{\pi(n)}\right)= \\
(-1)^{M} W_{(\alpha)(\dot{\beta}), \ldots,(\gamma)(\dot{\delta})}\left(x_{2}-x_{1}, \ldots, x_{n+1}-x_{n}\right)
\end{array}
$$


where $M$ is the number of the transpositions of the anti - commuting fields under the permutation $\pi$. In general case the distribution in the left - hand side of the equality (1.12) is another vacuum expectation value of the product of the quantum fields. This vacuum expectation value depends on the permutation $\pi$.

Lorentz invariant distributions are studied in the papers [3] - [5]. The description of Lorentz covariant tempered distributions with supports in the product of the closed upper light cones (1.7) is obtained in the paper [6]. The attempts to construct a non - trivial example of the distributions satisfying the properties of spectrality, Lorentz covariance and locality failed.

The aim of this paper is the mathematical statement of the properties of the vacuum expectation values of the quantum fields products. The distributions (1.4) for $n=1$ are described in the paper [6]. We suppose that the distributions (1.4) for $n>1$ are similar to the distributions (1.4) for $n=1$. Our requirements are connected with three groups: the group of the translations, Lorentz group and the group of the permutations of the natural numbers $1, \ldots, n+1$. The vacuum expectation values of the products of the quantum fields are the series of the distributions. If these series are finite, the vacuum expectation values of the products of the quantum fields satisfy the properties of spectrality, Lorentz covariance and locality. We define the convergence of the infinite series of the distributions in such a manner that the vacuum expectation values of the products of the quantum fields satisfy the properties of Lorentz covariance and locality. We propose a new asymptotic condition for the vacuum expectation values of the products of the quantum fields. We deal with the asymptotic quantum fields in an experiment. The asymptotic quantum fields may be considered free. The positivity property [2] should be applied to the asymptotic vacuum expectation values of the products of the quantum fields.

\section{Lorentz covariance and spectral condition}

The group $S U(2)$ is the maximal compact subgroup of the group $S L(2, \mathbf{C})$. It consists of the matrices from the group $S L(2, \mathbf{C})$ satisfying the equation $A A^{*}=\sigma^{0}$. Let us describe the irreducible representations of the group $S U(2)$. We consider the half - integers $l \in 1 / 2 \mathbf{Z}_{+}$, i.e. $l=0,1 / 2,1,3 / 2, \ldots$. We define the representation of the group $S U(2)$ on the space of the polynomials with degree less than or equal to $2 l$

$$
T_{l}(A) \phi(z)=\left(A_{12} z+A_{22}\right)^{2 l} \phi\left(\frac{A_{11} z+A_{21}}{A_{12} z+A_{22}}\right) .
$$

We consider a half - integer $n=-l,-l+1, \ldots, l-1, l$ and choose the polynomial basis

$$
\psi_{n}(z)=((l-n) !(l+n) !)^{-1 / 2} z^{l-n} .
$$

The definitions (2.1), (2.2) imply

$$
T_{l}(A) \psi_{n}(z)=\sum_{m=-l}^{l} \psi_{m}(z) t_{m n}^{l}(A)
$$

where the polynomial

$$
\sum_{j=-\infty}^{\infty} \frac{t_{m n}^{l}(A)=((l-m) !(l+m) !(l-n) !(l+n) !)^{1 / 2} \times}{\Gamma(j+1) \Gamma(l-m-j+1) \Gamma(m-n+j+1) \Gamma(l+n-j+1)}
$$


and $\Gamma(z)$ is the gamma - function. The function $(\Gamma(z))^{-1}$ equals zero for $z=0,-1,-2, \ldots$. Therefore the series (2.4) is a polynomial.

The relation (2.1) defines a representation of the group $S U(2)$. Thus the polynomial (2.4) defines a representation of the group $S U(2)$

$$
t_{m n}^{l}(A B)=\sum_{k=-l}^{l} t_{m k}^{l}(A) t_{k n}^{l}(B) .
$$

This $(2 l+1)$ - dimensional representation is irreducible ([7], Chapter III, Section 2.3).

The relations (2.4), (2.5) have an analytic continuation to the matrices from the group $S L(2, \mathbf{C})$. By making the change $j \rightarrow j+n-m$ of the summation variable in the equality (2.4) we have

$$
t_{m n}^{l}(A)=t_{n m}^{l}\left(A^{T}\right)
$$

Due to ([7], Chapter III, Section 8.3) we have

$$
\begin{array}{r}
t_{m_{1} n_{1}}^{l_{1}}(A) t_{m_{2} n_{2}}^{l_{2}}(A)=\sum_{l_{3} \in 1 / 2 \mathbf{Z}_{+}} \sum_{m_{3}, n_{3}=-l_{3}}^{l_{3}} \\
C\left(l_{1}, l_{2}, l_{3} ; m_{1}, m_{2}, m_{3}\right) C\left(l_{1}, l_{2}, l_{3} ; n_{1}, n_{2}, n_{3}\right) t_{m_{3} n_{3}}^{l_{3}}(A)
\end{array}
$$

for a matrix $A \in S U(2)$. The Clebsch - Gordan coefficient $C\left(l_{1}, l_{2}, l_{3} ; m_{1}, m_{2}, m_{3}\right)$ is not zero only if $m_{3}=m_{1}+m_{2}$ and the half - integers $l_{1}, l_{2}, l_{3} \in 1 / 2 \mathbf{Z}_{+}$satisfy the triangle condition: it is possible to construct a triangle with the sides of length $l_{1}, l_{2}, l_{3}$ and an integer perimeter $l_{1}+l_{2}+l_{3}$. It means that the half - integer $l_{3}$ is one of the half - integers $\left|l_{1}-l_{2}\right|,\left|l_{1}-l_{2}\right|+1, \ldots, l_{1}+l_{2}-1, l_{1}+l_{2}$. Let the half - integers $l_{1}, l_{2}, l_{3} \in 1 / 2 \mathbf{Z}_{+}$satisfy the triangle condition. Let the half - integers $m_{i}=-l_{i},-l_{i}+1, \ldots, l_{i}-1, l_{i}, i=1,2,3$, $m_{3}=m_{1}+m_{2}$. Then due to ([7], Chapter III, Section 8.3)

$$
\begin{gathered}
C\left(l_{1}, l_{2}, l_{3} ; m_{1}, m_{2}, m_{3}\right)=(-1)^{l_{1}-l_{3}+m_{2}}\left(2 l_{3}+1\right)^{1 / 2} \times \\
\left(\frac{\left(l_{1}+l_{2}-l_{3}\right) !\left(l_{1}+l_{3}-l_{2}\right) !\left(l_{2}+l_{3}-l_{1}\right) !\left(l_{3}-m_{3}\right) !\left(l_{3}+m_{3}\right) !}{\left(l_{1}+l_{2}+l_{3}+1\right) !\left(l_{1}-m_{1}\right) !\left(l_{1}+m_{1}\right) !\left(l_{2}-m_{2}\right) !\left(l_{2}+m_{2}\right) !}\right)^{1 / 2} \times \\
\sum_{j=0}^{l_{2}+l_{3}-l_{1}} \frac{\left.(-1)^{j}\left(l_{1}+m_{1}+j\right) !\right)\left(l_{2}+l_{3}-m_{1}-j\right) !}{j ! \Gamma\left(l_{3}-m_{3}-j+1\right) \Gamma\left(l_{1}-l_{2}+m_{3}+j+1\right)\left(l_{2}+l_{3}-l_{1}-j\right) !}
\end{gathered}
$$

Let $d A$ be the normalized Haar measure on the group $S U(2)$. Due to ([7], Chapter III, Section 8.3)

$$
\begin{array}{r}
C\left(l_{1}, l_{2}, l_{3} ; m_{1}, m_{2}, m_{3}\right) C\left(l_{1}, l_{2}, l_{3} ; n_{1}, n_{2}, n_{3}\right)= \\
\quad\left(2 l_{3}+1\right) \int_{S U(2)} d A t_{m_{1} n_{1}}^{l_{1}}(A) t_{m_{2} n_{2}}^{l_{2}}(A) \overline{t_{m_{3} n_{3}}^{l_{3}}(A)} .
\end{array}
$$

The coefficients of the polynomial (2.4) are real. By using the relations (2.6) and $A^{*}=A^{-1}$ we can rewrite the equality (2.9) as

$$
\begin{aligned}
& C\left(l_{1}, l_{2}, l_{3} ; m_{1}, m_{2}, m_{3}\right) C\left(l_{1}, l_{2}, l_{3} ; n_{1}, n_{2}, n_{3}\right)= \\
& \left(2 l_{3}+1\right) \int_{S U(2)} d A t_{m_{1} n_{1}}^{l_{1}}(A) t_{m_{2} n_{2}}^{l_{2}}(A) t_{n_{3} m_{3}}^{l_{3}}\left(A^{-1}\right) .
\end{aligned}
$$


If the half - integers $l_{1}, l_{2}, l_{3} \in 1 / 2 \mathbf{Z}_{+}$satisfy the triangle condition, then due to ([7], Chapter III, Section 8.3) we have

$$
C\left(l_{1}, l_{2}, l_{3} ; l_{1},-l_{2}, l_{1}-l_{2}\right)=\left(\frac{\left(2 l_{3}+1\right)\left(2 l_{1}\right) !\left(2 l_{2}\right) !}{\left(l_{1}+l_{2}-l_{3}\right) !\left(l_{1}+l_{2}+l_{3}+1\right) !}\right)^{1 / 2}
$$

Let us choose the half - integers $n_{1}=l_{1}, n_{2}=-l_{2}$ in the equality (2.10). Then the relation (2.10) and the invariance of the Haar measure $d A$ imply

$$
\begin{array}{r}
\sum_{n_{1}=-l_{1}}^{l_{1}} \sum_{n_{2}=-l_{2}}^{l_{2}} t_{m_{1} n_{1}}^{l_{1}}(A) t_{m_{2} n_{2}}^{l_{2}}(A) C\left(l_{1}, l_{2}, l_{3} ; n_{1}, n_{2}, m_{3}\right)= \\
\sum_{n_{3}=-l_{3}}^{l_{3}} C\left(l_{1}, l_{2}, l_{3} ; m_{1}, m_{2}, n_{3}\right) t_{n_{3} m_{3}}^{l_{3}}(A) .
\end{array}
$$

The substitution of the matrix (1.8) into the equality (2.12) and the equality (2.6) yield

$$
\begin{array}{r}
\sum_{n_{1}=-l_{1}}^{l_{1}} \sum_{n_{2}=-l_{2}}^{l_{2}} t_{n_{1} m_{1}}^{l_{1}}(A) t_{n_{2} m_{2}}^{l_{2}}(A) C\left(l_{1}, l_{2}, l_{3} ; n_{1}, n_{2}, m_{3}\right)= \\
\sum_{n_{3}=-l_{3}}^{l_{3}} C\left(l_{1}, l_{2}, l_{3} ; m_{1}, m_{2}, n_{3}\right) t_{m_{3} n_{3}}^{l_{3}}(A) .
\end{array}
$$

The relations (2.12) (2.13) have an analytic continuation to any matrix from the group $S L(2, \mathbf{C})$.

For any natural numbers $m, n$ and the half - integers $l_{1}, \ldots, l_{n+1}, l_{1}, \ldots, i_{n+1} \in 1 / 2 \mathbf{Z}_{+} ; m_{i}=$ $-l_{i},-l_{i}+1, \ldots, l_{i}-1, l_{i}, \dot{m}_{i}=-\dot{l}_{i},-\dot{l}_{i}+1, \ldots, \dot{l}_{i}-1, \dot{l}_{i}, i=1, \ldots, n+1$, we consider the set of the tempered distributions

$$
F_{m_{1}, \ldots, m_{n+1} ; \dot{m}_{1}, \ldots, \dot{m}_{n+1}}^{l_{1}, \ldots, l_{n+1} ; i_{1}, \ldots, i_{n+1}}\left(x_{1}, \ldots, x_{m}\right) \in S^{\prime}\left(\mathbf{R}^{4 m}\right) .
$$

This set is called a Lorentz covariant distribution if for any matrix $A \in S L(2, \mathbf{C})$

$$
\begin{array}{r}
F_{m_{1}, \ldots, m_{n+1} ; \dot{m}_{1}, \ldots, \dot{m}_{n+1}}^{l_{1}, \ldots, l_{n+1} ; i_{1}, \ldots, i_{n+1}}\left(A \tilde{x}_{1} A^{*}, \ldots, A \tilde{x}_{m} A^{*}\right)=\sum_{k_{1}=-l_{1}}^{l_{1}} \ldots \sum_{k_{n+1}=-l_{n+1}}^{l_{n+1}} \sum_{\dot{k}_{1}=-i_{1}}^{l_{1}} \ldots \sum_{\dot{k}_{n+1}=-i_{n+1}}^{i_{n+1}} \\
\left(\prod_{i=1}^{n+1} t_{m_{i} k_{i}}^{l_{i}}(A) t_{\dot{m}_{i} \dot{k}_{i}}^{i_{i}}(\bar{A})\right) F_{k_{1}, \ldots, k_{n+1} ; k_{1}, \ldots, \dot{k}_{n+1}}^{l_{1}, \ldots, l_{n+1} ; i_{1}, \ldots, i_{n+1}}\left(\tilde{x}_{1}, \ldots, \tilde{x}_{m}\right)
\end{array}
$$

where $2 \times 2$ - matrix $\tilde{x}$ is given by the relation $(1.10)$. The half - integers $l_{1}, \ldots, l_{n+1}, \dot{l}_{1}, \ldots, \dot{l}_{n+1}$ in the relation (2.14) are not arbitrary. Let us choose the matrix $A=-\sigma^{0}$ in the equality (2.14). For this matrix

$$
A \tilde{x}_{j} A^{*}=\tilde{x}_{j}, j=1, \ldots, m \text {. }
$$

The definition (2.4) implies

$$
t_{m_{j} k_{j}}^{l_{j}}\left(-\sigma^{0}\right)=(-1)^{2 l_{j}} \delta_{m_{j} k_{j}} .
$$

Hence the equality (2.14) is valid for the matrix $A=-\sigma^{0}$ if

$$
(-1)^{2\left(l_{1}+\cdots+l_{n+1}+i_{1}+\cdots+i_{n+1}\right)}=1
$$


i.e. the sum $l_{1}+\cdots+l_{n+1}+i_{1}+\cdots+i_{n+1}$ is an integer. This condition is supposed below.

Let a tempered distribution $F(x) \in S^{\prime}\left(\mathbf{R}^{4}\right)$ have a support in the closed upper light cone (1.7). Due to the paper [8] there is a natural number $q$ such that

$$
\begin{gathered}
F(x)=\left(\partial_{x}, \partial_{x}\right)^{q} f(x) \\
\left(\partial_{x}, \partial_{x}\right)=\left(\frac{\partial}{\partial x^{0}}\right)^{2}-\sum_{k=1}^{3}\left(\frac{\partial}{\partial x^{k}}\right)^{2}
\end{gathered}
$$

where a differentiable function $f(x)$ with a support in the closed upper light cone (1.7) is polynomial bounded.

Let us introduce the step function

$$
\theta(x)= \begin{cases}1, & x \geq 0 \\ 0, & x<0\end{cases}
$$

Due to ([6], Proposition) any tempered distribution

$$
F_{m_{1}, m_{2} ; \dot{m}_{1}, \dot{m}_{2}}^{l_{1}, l_{2} ; i_{1}, i_{2}}(x) \in S^{\prime}\left(\mathbf{R}^{4}\right)
$$

with a support in the closed upper light cone (1.7) satisfying the covariance relation (2.14) for $m=n=1$ has the following form

$$
\begin{array}{r}
\int d^{4} x F_{m_{1}, m_{2} ; \dot{m}_{1}, \dot{m}_{2}}^{l_{1}, l_{2} ; \dot{l}_{1}, i_{2}}(x) \phi(x)= \\
\sum_{l_{3} \in 1 / 2 \mathbf{Z}_{+}} \sum_{m_{3}, \dot{m}_{3}=-l_{3}}^{l_{3}} C\left(l_{1}, l_{2}, l_{3} ; m_{1}, m_{2}, m_{3}\right) C\left(\dot{l}_{1}, \dot{l}_{2}, l_{3} ; \dot{m}_{1}, \dot{m}_{2}, \dot{m}_{3}\right) \times \\
\int d^{4} x \theta\left(x^{0}\right) \theta((x, x)) t_{m_{3} \dot{m}_{3}}^{l_{3}}(\tilde{x}) f^{l_{1}, l_{2}, l_{3} ; \dot{l}_{1}, i_{2}}\left((x, x)^{1 / 2}\right)\left(\partial_{x}, \partial_{x}\right)^{q} \phi(x)
\end{array}
$$

where a test function $\phi(x) \in S\left(\mathbf{R}^{4}\right) ; q$ is a natural number; the Clebsch - Gordan coefficient $C\left(l_{1}, l_{2}, l_{3} ; m_{1}, m_{2}, m_{3}\right)$ is given by the relation $(2.8) ; 2 \times 2$ - matrix $\tilde{x}$ is given by the relation (1.10); the polynomial $t_{m n}^{l}(A)$ is given by the relation (2.4); the differentiable function $f^{l_{1}, l_{2}, l_{3} ; i_{1}, l_{2}}(s)$ with a support in the positive semi - axis is polynomial bounded.

Let us consider a tempered distribution with a support in the cone (1.7) satisfying the covariance relation (2.14) for $m=1, n=0$. This case corresponds to the equality (2.18) for $l_{2}=\dot{l}_{2}=0, m_{2}=\dot{m}_{2}=0$. The relation (2.8) implies

$$
C\left(l_{1}, 0, l_{3} ; m_{1}, 0, m_{3}\right)=\delta_{l_{1} l_{3}} \delta_{m_{1} m_{3}} .
$$

The vacuum expectation value of the product of two quantum field is the Fourier transform of the distribution (2.18). Let us represent the distribution (2.18) in the form suitable for taking Fourier transform.

By making use of the matrices (1.9) as the coefficients we define $2 \times 2$ - matrix

$$
\tilde{\partial}_{x}=\sum_{\mu=0}^{3} \eta^{\mu \mu} \sigma^{\mu} \frac{\partial}{\partial x^{\mu}}
$$

where $\eta^{00}=-\eta^{11}=-\eta^{22}=-\eta^{33}=1$. We insert the matrix (2.19) into the polynomial (2.4) and obtain the differential operator $t_{m n}^{l}\left(\tilde{\partial}_{x}\right)$. 
Lemma 2.1. Let $f(s)$ be $2 l$ times differentiable function. Then

$$
t_{m n}^{l}\left(\tilde{\partial}_{x}\right) f((x, x))=\left.t_{m n}^{l}(\tilde{x})\left(2 \frac{d}{d s}\right)^{2 l} f(s)\right|_{s=(x, x)}
$$

Proof. The definitions (1.9), (2.4), (2.19) imply

$$
\begin{array}{r}
t_{m n}^{l}\left(\tilde{\partial}_{x}\right) f((x, x))=((l-m) !(l+m) !(l-n) !(l+n) !)^{1 / 2} \times \\
\sum_{j=-\infty}^{\infty}(\Gamma(j+1) \Gamma(l-m-j+1) \Gamma(m-n+j+1) \Gamma(l+n-j+1))^{-1} \times \\
\left(\frac{\partial}{\partial x^{0}}-\frac{\partial}{\partial x^{3}}\right)^{l-m-j}\left(-\frac{\partial}{\partial x^{1}}+i \frac{\partial}{\partial x^{2}}\right)^{j} \times \\
\left(\left.\left(x^{1}+i x^{2}\right)^{m-n+j}\left(x^{0}-x^{3}\right)^{l+n-j}\left(2 \frac{d}{d s}\right)^{l+m} f(s)\right|_{s=(x, x)}\right) .
\end{array}
$$

By using Leibniz rule we have

$$
\begin{array}{r}
\left(\frac{\partial}{\partial x^{0}}-\frac{\partial}{\partial x^{3}}\right)^{l-m-j}\left(-\frac{\partial}{\partial x^{1}}+i \frac{\partial}{\partial x^{2}}\right)^{j} \times \\
\left(\left.\left(x^{1}+i x^{2}\right)^{m-n+j}\left(x^{0}-x^{3}\right)^{l+n-j}\left(2 \frac{d}{d s}\right)^{l+m} f(s)\right|_{s=(x, x)}\right)= \\
\sum_{p=-\infty}^{\infty} \sum_{q=-\infty}^{\infty} \frac{\Gamma(l-m-j+1) \Gamma(j+1)}{\Gamma(p+1) \Gamma(l-m-j-p+1) \Gamma(q+1) \Gamma(j-q+1)} \times \\
\left(\left(\frac{\partial}{\partial x^{0}}-\frac{\partial}{\partial x^{3}}\right)^{p}\left(x^{0}-x^{3}\right)^{l+n-j}\right)\left(\left(-\frac{\partial}{\partial x^{1}}+i \frac{\partial}{\partial x^{2}}\right)^{q}\left(x^{1}+i x^{2}\right)^{m-n+j}\right) \times \\
\left.\left(\frac{\partial}{\partial x^{0}}-\frac{\partial}{\partial x^{3}}\right)^{l-m-j-p}\left(-\frac{\partial}{\partial x^{1}}+i \frac{\partial}{\partial x^{2}}\right)^{j-q}\left(2 \frac{d}{d s}\right)^{l+m} f(s)\right|_{s=(x, x)} .
\end{array}
$$

By calculating the derivatives in the right - hand side of the equality (2.22) we can rewrite the equality (2.21) in the form

$$
\begin{array}{r}
t_{m n}^{l}\left(\tilde{\partial}_{x}\right) f((x, x))=((l-m) !(l+m) !(l-n) !(l+n) !)^{1 / 2} \times \\
\sum_{j=-\infty}^{\infty} \sum_{p=-\infty}^{\infty} \sum_{q=-\infty}^{\infty}(-1)^{q} 2^{p+q}(\Gamma(p+1) \Gamma(q+1))^{-1} \times \\
(\Gamma(j-q+1) \Gamma(l-m-j-p+1) \Gamma(m-n+j-q+1) \Gamma(l+n-j-p+1))^{-1} \times \\
\left(x^{0}+x^{3}\right)^{l-m-j-p}\left(x^{0}-x^{3}\right)^{l+n-j-p}\left(x^{1}+i x^{2}\right)^{m-n+j-q}\left(x^{1}-i x^{2}\right)^{j-q} \times \\
\left.\left(2 \frac{d}{d s}\right)^{2 l-p-q} f(s)\right|_{s=(x, x)} .
\end{array}
$$

By making the changes $j \rightarrow j+q, p \rightarrow p-q$ of the summation variables we have

$$
\begin{array}{r}
t_{m n}^{l}\left(\tilde{\partial}_{x}\right) f((x, x))=((l-m) !(l+m) !(l-n) !(l+n) !)^{1 / 2} \times \\
\sum_{j=-\infty}^{\infty} \sum_{p=-\infty}^{\infty} \sum_{q=-\infty}^{\infty}(-1)^{q} 2^{p}(\Gamma(p-q+1) \Gamma(q+1))^{-1} \times
\end{array}
$$




$$
\begin{gathered}
(\Gamma(j+1) \Gamma(l-m-j-p+1) \Gamma(m-n+j+1) \Gamma(l+n-j-p+1))^{-1} \times \\
\left.\left(x^{0}+x^{3}\right)^{l-m-j-p}\left(x^{0}-x^{3}\right)^{l+n-j-p}\left(x^{1}+i x^{2}\right)^{m-n+j}\left(x^{1}-i x^{2}\right)^{j}\left(2 \frac{d}{d s}\right)^{2 l-p} f(s)\right|_{s=(x, x)} .
\end{gathered}
$$

The identity

$$
\sum_{q=0}^{p}(-1)^{q} \frac{\Gamma(p+1)}{\Gamma(p-q+1) \Gamma(q+1)}=(1-1)^{p}
$$

is valid. For $p \geq 1$ both sides of the equality (2.25) are equal to zero. For $p=0$ the left hand side of the equality (2.25) is equal to 1 . By making use of the equality (2.25) we can rewrite the equality (2.24) in the form (2.20). The lemma is proved.

Let us define

$$
f^{(-2 l)}(s)=\theta(s) \int_{0}^{s} d t \frac{(s-t)^{2 l-1}}{(2 l-1) !} f\left(t^{1 / 2}\right), 2 l \geq 1, f^{(0)}(s)=\theta(s) f\left(s^{1 / 2}\right) .
$$

By using the definitions (2.26) we have

$$
\left(\frac{d}{d s}\right)^{2 l} f^{(-2 l)}(s)=\theta(s) f\left(s^{1 / 2}\right) .
$$

The equalities (2.20), (2.27) imply

$$
t_{m n}^{l}(\tilde{x}) \theta((x, x)) f\left((x, x)^{1 / 2}\right)=2^{-2 l} t_{m n}^{l}\left(\tilde{\partial}_{x}\right) f^{(-2 l)}((x, x)) .
$$

If the function $f(s)$ is polynomial bounded, then the function (2.26) is also polynomial bounded. If the function $f(s)$ is continuous and has the support in the positive semi - axis, then the function (2.26) is also continuous and has the support in the positive semi - axis. The function (2.26) is $2 l$ times differentiable and its first $2 l$ derivatives are equal to zero at the point $s=0$. Therefore the equality (2.28) implies

$$
\begin{array}{r}
\theta\left(x^{0}\right) \theta((x, x)) t_{m_{3} \dot{m}_{3}}^{l_{3}}(\tilde{x}) f^{l_{1}, l_{2}, l_{3} ; i_{1}, i_{2}}\left((x, x)^{1 / 2}\right)= \\
t_{m_{3} \dot{m}_{3}}^{l_{3}}\left(\tilde{\partial}_{x}\right)\left(\theta\left(x^{0}\right) 2^{-2 l_{3}}\left(f^{l_{1}, l_{2}, l_{3} ; i_{1}, i_{2}}\right)^{\left(-2 l_{3}\right)}((x, x))\right) .
\end{array}
$$

The substitution of the equality (2.29) into the relation (2.18) gives the following form of any tempered distribution

$$
F_{m_{1}, m_{2} ; \dot{m}_{1}, \dot{m}_{2}}^{l_{1}, l_{2} ; i_{1}, i_{2}}(x) \in S^{\prime}\left(\mathbf{R}^{4}\right)
$$

with a support in the closed upper light cone (1.7) satisfying the covariance relation (2.14) for $m=n=1$

$$
\begin{array}{r}
\int d^{4} x F_{m_{1}, m_{2} ; \dot{m}_{1}, \dot{m}_{2}}^{l_{1}, l_{2} ; \dot{l}_{1}, i_{2}}(x) \phi(x)= \\
\sum_{l_{3} \in 1 / 2 \mathbf{Z}_{+}} \sum_{m_{3}, \dot{m}_{3}=-l_{3}}^{l_{3}} C\left(l_{1}, l_{2}, l_{3} ; m_{1}, m_{2}, m_{3}\right) C\left(\dot{l}_{1}, \dot{l}_{2}, l_{3} ; \dot{m}_{1}, \dot{m}_{2}, \dot{m}_{3}\right) \times \\
\int d^{4} x \theta\left(x^{0}\right) 2^{-2 l_{3}}\left(f^{l_{1}, l_{2}, l_{3} ; \dot{l}_{1}, \dot{l}_{2}}\right)^{\left(-2 l_{3}\right)}((x, x))\left(\partial_{x}, \partial_{x}\right)^{q} t_{m_{3} \dot{m}_{3}}^{l_{3}}\left(-\tilde{\partial}_{x}\right) \phi(x)
\end{array}
$$

where a test function $\phi(x) \in S\left(\mathbf{R}^{4}\right) ; q$ is a natural number; the Clebsch - Gordan coefficient $C\left(l_{1}, l_{2}, l_{3} ; m_{1}, m_{2}, m_{3}\right)$ is given by the relation $(2.8) ; 2 \times 2-$ matrix $\tilde{\partial}_{x}$ is given by the 
relation (2.19); the polynomial $t_{m n}^{l}(A)$ is given by the relation (2.4); the continuous function $\left(f^{l_{1}, l_{2}, l_{3} ; i_{1}, i_{2}}\right)^{\left(-2 l_{3}\right)}(s)$ with a support in the positive semi - axis is polynomial bounded.

Let us consider the tempered distributions of the form

$$
\begin{array}{r}
\int d^{4 m} x F_{m_{1}, \ldots, m_{n+1} ; \dot{m}_{1}, \ldots, \dot{m}_{n+1}}^{l_{1}, \ldots, l_{n+1} ; i_{1}, \ldots, i_{n+1}}\left(x_{1}, \ldots, x_{m}\right) \phi\left(x_{1}, \ldots, x_{m}\right)= \\
\sum_{l_{n+2}, \ldots, l_{n+m+1} \in 1 / 2 \mathbf{Z}_{+} m_{s}, \dot{m}_{s}=-l_{s},-l_{s}+1, \ldots, l_{s}-1, l_{s}, s=n+2, \ldots, n+m+1} \\
\int d^{4 m} x f_{m_{1}, \ldots, m_{n+m+1} ; \dot{m}_{1}, \ldots, \dot{m}_{n+m+1}}^{l_{1}, \ldots, l_{n+m+1} ; \dot{l}_{1}, \ldots, i_{n+1}}\left(\left(x_{1}, x_{1}\right), \ldots,\left(x_{m}, x_{m}\right)\right) \times \\
\left(\prod_{j=1}^{m}\left(\theta\left(x_{j}^{0}\right)\left(\partial_{x_{j}}, \partial_{x_{j}}\right)^{q} t_{m_{n+1+j} l_{n+1+j} \dot{m}_{n+1+j}}^{l_{n+1}}\left(-\tilde{\partial}_{x_{j}}\right)\right)\right) \phi\left(x_{1}, \ldots, x_{m}\right)
\end{array}
$$

where a test function $\phi\left(x_{1}, \ldots, x_{m}\right) \in S\left(\mathbf{R}^{4 m}\right) ; q$ is a natural number; $2 \times 2$ - matrix $\tilde{\partial}_{x}$ is given by the relation (2.19); the polynomial $t_{m n}^{l}(A)$ is given by the relation $(2.4)$; the continuous function

$$
f_{m_{1}, \ldots, m_{n+m+1} ; \dot{m}_{1}, \ldots, \dot{m}_{n+m+1}}^{l_{1}, \ldots, l_{n+m+1} ; i_{1}, \ldots, i_{n+1}}\left(s_{1}, \ldots, s_{m}\right)
$$

with a support in the product of the positive semi - axes is polynomial bounded; the sums over the summation variables $l_{n+2}, \ldots, l_{n+m+1}$ are finite. The distribution (2.31) belongs to the space $S^{\prime}\left(\mathbf{R}^{4 m}\right)$. The sum over the summation variable $l_{3}$ in the relation (2.30) is finite due to the triangle condition for the Clebsch - Gordan coefficients.

For any numbers $m, n \in \mathbf{Z}_{+}$we define the generalized Clebsch - Gordan coefficient

$$
\begin{array}{r}
C\left(l_{1}, \ldots, l_{m+2} ; l_{m+3}, \ldots, l_{m+n+4} ; j_{1}, \ldots, j_{m+n+1} ; m_{1}, \ldots, m_{m+2} ; m_{m+3}, \ldots, m_{m+n+4}\right)= \\
C\left(l_{1}, l_{2}, j_{1} ; m_{1}, m_{2}, k_{1}\right) \times \\
k_{s}=-j_{s},-j_{s}+1, \ldots, j_{s}-1, j_{s}, s=1, \ldots, m+n+1 \\
\left(\prod_{s=1}^{m} C\left(j_{s}, l_{s+2}, j_{s+1} ; k_{s}, m_{s+2}, k_{s+1}\right)\right)\left(\prod_{s=m+1}^{m+n} C\left(j_{s+1}, l_{s+2}, j_{s} ; k_{s+1}, m_{s+2}, k_{s}\right)\right) \times \\
C\left(l_{m+n+4}, l_{m+n+3}, j_{m+n+1} ; m_{m+n+4}, m_{m+n+3}, k_{m+n+1}\right)
\end{array}
$$

where the half - integers $l_{1}, \ldots, l_{m+n+4}, j_{1}, \ldots, j_{m+n+1} \in 1 / 2 \mathbf{Z}_{+}$and $m_{i}=-l_{i},-l_{i}+1, \ldots, l_{i}-$ $1, l_{i}, i=1, \ldots, m+n+4$. The definition (2.32) and the relations (2.12), (2.13) imply for any matrix $A \in S L(2, \mathbf{C})$

$$
\begin{array}{r}
\sum_{n_{s}=-l_{s},-l_{s}+1, \ldots, l_{s}-1, l_{s}, s=1, \ldots, m+2}\left(\prod_{i=1}^{m+2} t_{m_{i}, n_{i}}^{l_{i}}(A)\right) \times \\
C\left(l_{1}, \ldots, l_{m+2} ; l_{m+3}, \ldots, l_{m+n+4} ; j_{1}, \ldots, j_{m+n+1} ; n_{1}, \ldots, n_{m+2} ; m_{m+3}, \ldots, m_{m+n+4}\right) \\
\sum_{n_{s}=-l_{s},-l_{s}+1, \ldots, l_{s}-1, l_{s}, s=m+3, \ldots, m+n+4}\left(\prod_{i=m+3}^{m+n+4} t_{n_{i}, m_{i}}^{l_{i}}(A)\right) \times \\
C\left(l_{1}, \ldots, l_{m+2} ; l_{m+3}, \ldots, l_{m+n+4} ; j_{1}, \ldots, j_{m+n+1} ; m_{1}, \ldots, m_{m+2} ; n_{m+3}, \ldots, n_{m+n+4}\right), \\
\sum_{n_{s}=-l_{s},-l_{s}+1, \ldots, l_{s}-1, l_{s}, s=1, \ldots, m+2}\left(\prod_{i=1}^{m+2} t_{n_{i}, m_{i}}^{l_{i}}(A)\right) \times \\
C\left(l_{1}, \ldots, l_{m+2} ; l_{m+3}, \ldots, l_{m+n+4} ; j_{1}, \ldots, j_{m+n+1} ; n_{1}, \ldots, n_{m+2} ; m_{m+3}, \ldots, m_{m+n+4}\right)
\end{array}
$$




$$
\begin{gathered}
\sum_{n_{s}=-l_{s},-l_{s}+1, \ldots, l_{s}-1, l_{s}, s=m+3, \ldots, m+n+4}\left(\prod_{i=m+3}^{m+n+4} t_{m_{i}, n_{i}}^{l_{i}}(A)\right) \times \\
C\left(l_{1}, \ldots, l_{m+2} ; l_{m+3}, \ldots, l_{m+n+4} ; j_{1}, \ldots, j_{m+n+1} ; m_{1}, \ldots, m_{m+2} ; n_{m+3}, \ldots, n_{m+n+4}\right) .
\end{gathered}
$$

We will consider in the following sections the distributions $(2.31)$ for the case $m=\frac{1}{2} n(n+1)$ only.

Theorem 2.2. Let the distributions (2.31) for $m=n=1$ satisfy the covariance relation (2.14), then

$$
f_{m_{1}, m_{2}, m_{3} ; \dot{m}_{1}, \dot{m}_{2}, \dot{m}_{3}}^{l_{1}, l_{2}, l_{3} ; \dot{l}_{1}, i_{2}}\left(s_{1}\right)=f^{l_{1}, l_{2}, l_{3} ; i_{1}, i_{2}}\left(s_{1}\right) C\left(l_{1}, l_{2}, l_{3} ; m_{1}, m_{2}, m_{3}\right) C\left(\dot{l}_{1}, \dot{l}_{2}, l_{3} ; \dot{m}_{1}, \dot{m}_{2}, \dot{m}_{3}\right)
$$

where a continuous function $f^{l_{1}, l_{2}, l_{3} ; i_{1}, i_{2}}\left(s_{1}\right)$ with a support in the positive semi - axis is polynomial bounded; the Clebsch - Gordan coefficient $C\left(l_{1}, l_{2}, l_{3} ; m_{1}, m_{2}, m_{3}\right)$ is given by the relation $(2.8)$.

Let the distributions (2.31) for $m>2, n>1$ satisfy the covariance relation (2.14), then

$$
\begin{aligned}
& f_{m_{1}, \ldots, m_{n+m+1} ; \dot{m}_{1}, \ldots, \dot{m}_{n+m+1}}^{l_{1}, \ldots, l_{n+m+1} ; \dot{l}_{1}, \ldots, i_{n+1}}\left(s_{1}, \ldots, s_{m}\right)= \\
& \sum_{j_{1}, \ldots, j_{n+m-2} ; j_{1}^{\prime}, \ldots, j_{n+m-2}^{\prime} \in 1 / 2 \mathbf{Z}_{+}} f_{j_{1}, \ldots, j_{n+m-2} ; j_{1}^{\prime}, \ldots, j_{n+m-2}^{\prime}}^{l_{1}, \ldots, l_{n+m+1} ; i_{1}, \ldots, i_{n+1}}\left(s_{1}, \ldots, s_{m}\right) \times \\
& C\left(l_{1}, \ldots, l_{n+1} ; l_{n+2}, \ldots, l_{m+n+1} ; j_{1}, \ldots, j_{m+n-2} ; m_{1}, \ldots, m_{n+1} ; m_{n+2}, \ldots, m_{m+n+1}\right) \times \\
& C\left(\dot{l}_{1}, \ldots, \dot{l}_{n+1} ; l_{n+2}, \ldots, l_{m+n+1} ; j_{1}^{\prime}, \ldots, j_{m+n-2}^{\prime} ; \dot{m}_{1}, \ldots, \dot{m}_{n+1} ; \dot{m}_{n+2}, \ldots, \dot{m}_{m+n+1}\right)
\end{aligned}
$$

where a continuous function

$$
f_{j_{1}, \ldots, j_{n+m-2} ; j_{1}^{\prime}, \ldots, j_{n+m-2}^{\prime}}^{l_{1}, \ldots, l_{n+m+1} ; i_{1}, \ldots, i_{n+1}}\left(s_{1}, \ldots, s_{m}\right)
$$

with a support in the product of the positive semi - axes is polynomial bounded; the generalized Clebsch - Gordan coefficient

$$
C\left(l_{1}, \ldots, l_{m+2} ; l_{m+3}, \ldots, l_{m+n+4} ; j_{1}, \ldots, j_{m+n+1} ; m_{1}, \ldots, m_{m+2} ; m_{m+3}, \ldots, m_{m+n+4}\right)
$$

is given by the relation (2.32).

Proof. The covariance relation (2.14) may be rewritten as

$$
\begin{aligned}
& F_{m_{1}, \ldots, m_{n+1} ; \dot{m}_{1}, \ldots, \dot{m}_{n+1}}^{l_{1}, \ldots, l_{n+1} ; i_{1}, \ldots, i_{n+1}}\left(\tilde{x}_{1}, \ldots, \tilde{x}_{m}\right)= \\
& \sum_{k_{s}=-l_{s},-l_{s}+1, \ldots, l_{s}-1, l_{s}, s=1, \ldots, n+1} \sum_{\dot{k}_{s}=-i_{s},-i_{s}+1, \ldots, i_{s}-1, i_{s}, s=1, \ldots, n+1} \\
& \left(\prod_{i=1}^{n+1} t_{m_{i} k_{i}}^{l_{i}}\left(A^{-1}\right) t_{\dot{m}_{i} \dot{k}_{i}}^{i_{i}}\left(\bar{A}^{-1}\right)\right) F_{k_{1}, \ldots, k_{n+1} ; \dot{k}_{1}, \ldots, \dot{k}_{n+1}}^{l_{1}, \ldots, l_{n+1} \dot{l}_{1}, \ldots, i_{n+1}}\left(A \tilde{x}_{1} A^{*}, \ldots, A \tilde{x}_{m} A^{*}\right) .
\end{aligned}
$$

Now the equality (2.31) and the relations (2.5), (2.6) imply

$$
\begin{array}{r}
\int d^{4 m} x F_{m_{1}, \ldots, m_{n+1} ; \dot{m}_{1}, \ldots, \dot{m}_{n+1}}^{l_{1}, \ldots, l_{n+1} ; \dot{l}_{1}, \ldots, i_{n+1}}\left(\tilde{x}_{1}, \ldots, \tilde{x}_{m}\right) \phi\left(x_{1}, \ldots, x_{m}\right)= \\
\sum_{l_{n+2}, \ldots, l_{n+m+1} \in 1 / 2 \mathbf{Z}_{+}} \sum_{k_{s}=-l_{s},-l_{s}+1, \ldots, l_{s}-1, l_{s}, s=1, \ldots, n+1} \sum_{\dot{k}_{s}=-i_{s},-i_{s}+1, \ldots, i_{s}-1, i_{s}, s=1, \ldots, n+1}
\end{array}
$$




$$
\begin{gathered}
\sum_{m_{s} \dot{m}_{s}, k_{s}, \dot{k}_{s}=-l_{s},-l_{s}+1, \ldots, l_{s}-1, l_{s}, s=n+2, \ldots, n+m+1} \\
\left(\prod_{i=1}^{n+1} t_{m_{i} k_{i}}^{l_{i}}\left(A^{-1}\right) t_{\dot{m}_{i} \dot{k}_{i}}^{\dot{i}_{i}}\left(\bar{A}^{-1}\right)\right)\left(\prod_{j=n+2}^{n+m+1} t_{m_{j} k_{j}}^{l_{j}}(A) t_{\dot{m}_{j} \dot{k}_{j}}^{i_{j}}(\bar{A})\right) \times \\
\int d^{4 m} x f_{k_{1}, \ldots, k_{n+m+1} ; \dot{k}_{1}, \ldots, \dot{k}_{n+m+1}}^{l_{1}, \ldots, l_{n+m+1} ; \dot{l}_{1}, \ldots, i_{n+1}}\left(\left(x_{1}, x_{1}\right), \ldots,\left(x_{m}, x_{m}\right)\right) \times \\
\left(\prod_{j=1}^{m} \theta\left(x_{j}^{0}\right)\left(\partial_{x_{j}}, \partial_{x_{j}}\right)^{q} t_{k_{n+1+j}, \dot{k}_{n+1+j}}^{l_{n+1+j}}\left(-\tilde{\partial}_{x_{j}}\right)\right) \phi\left(x_{1}, \ldots, x_{m}\right)
\end{gathered}
$$

for any matrix $A \in S L(2, \mathbf{C})$.

The sums (2.38) over the half - integers $l_{n+2}, \ldots, l_{n+m+1}$ are finite. Hence the right - hand side of the equality (2.38) is a polynomial of the matrix elements of the matrices $A, \bar{A}$. Let us choose the matrix from the group $S L(2, \mathbf{C})$

$$
\begin{array}{r}
A=\exp \left\{\frac{1}{4}\left(\phi_{1}^{\prime}+i \phi_{1}\right) \sigma^{3}\right\} \exp \left\{\frac{1}{4}\left(\theta_{1}^{\prime}+i \theta_{1}\right) \sigma^{1}\right\} \exp \left\{\frac{1}{4}\left(\psi_{1}^{\prime}+i \psi_{1}\right) \sigma^{3}\right\} \times \\
\exp \left\{\frac{1}{4}\left(\phi_{2}^{\prime}-i \phi_{2}\right) \sigma^{3}\right\} \exp \left\{\frac{1}{4}\left(\theta_{2}^{\prime}-i \theta_{2}\right) \sigma^{1}\right\} \exp \left\{\frac{1}{4}\left(\psi_{2}^{\prime}-i \psi_{2}\right) \sigma^{3}\right\} .
\end{array}
$$

Due to the definition (1.9) the matrix elements of the matrices $\sigma^{1}, \sigma^{3}$ are real. Therefore

$$
\begin{array}{r}
\bar{A}=\exp \left\{\frac{1}{4}\left(\phi_{1}^{\prime}-i \phi_{1}\right) \sigma^{3}\right\} \exp \left\{\frac{1}{4}\left(\theta_{1}^{\prime}-i \theta_{1}\right) \sigma^{1}\right\} \exp \left\{\frac{1}{4}\left(\psi_{1}^{\prime}-i \psi_{1}\right) \sigma^{3}\right\} \times \\
\exp \left\{\frac{1}{4}\left(\phi_{2}^{\prime}+i \phi_{2}\right) \sigma^{3}\right\} \exp \left\{\frac{1}{4}\left(\theta_{2}^{\prime}+i \theta_{2}\right) \sigma^{1}\right\} \exp \left\{\frac{1}{4}\left(\psi_{2}^{\prime}+i \psi_{2}\right) \sigma^{3}\right\} .
\end{array}
$$

The matrices $(2.39),(2.40)$ have the analytic continuation to the parameters $\phi_{k}^{\prime}=i \phi_{k}, \theta_{k}^{\prime}=$ $\theta_{k}, \psi_{k}^{\prime}=i \psi_{k}, k=1,2$ :

$$
\begin{array}{r}
A=u\left(\phi_{1}, \theta_{1}, \psi_{1}\right), \bar{A}=u\left(\phi_{2}, \theta_{2}, \psi_{2}\right), \\
u(\phi, \theta, \psi)=\exp \left\{i \frac{\phi}{2} \sigma^{3}\right\} \exp \left\{i \frac{\theta}{2} \sigma^{1}\right\} \exp \left\{i \frac{\psi}{2} \sigma^{3}\right\} .
\end{array}
$$

Due to the ([7], Chapter III, Section 6.1) the normalized Haar measure on the group $S U(2)$

$$
d u(\phi, \theta, \psi)=\left(16 \pi^{2}\right)^{-1} \sin \theta d \theta d \phi d \psi .
$$

We insert the matrices (2.41) into the relation (2.38) and integrate the obtained relation over both groups $S U(2)$ with the measures (2.42).

$$
\begin{aligned}
& \int d^{4 m} x F_{m_{1}, \ldots, m_{n+1} ; \dot{m}_{1}, \ldots, \dot{m}_{n+1}}^{l_{1}, \ldots, l_{n+1} ; \dot{l}_{1}, \ldots, i_{n+1}}\left(\tilde{x}_{1}, \ldots, \tilde{x}_{m}\right) \phi\left(x_{1}, \ldots, x_{m}\right)= \\
& \sum_{l_{n+2}, \ldots, l_{n+m+1} \in 1 / 2 \mathbf{Z}_{+}} \sum_{k_{s}=-l_{s},-l_{s}+1, \ldots, l_{s}-1, l_{s}, s=1, \ldots, n+1} \sum_{\dot{k}_{s}=-i_{s},-i_{s}+1, \ldots, i_{s}-1, i_{s}, s=1, \ldots, n+1} \\
& \sum_{m_{s} \dot{m}_{s}, k_{s}, \dot{k}_{s}=-l_{s},-l_{s}+1, \ldots, l_{s}-1, l_{s}, s=n+2, \ldots, n+m+1} \int_{S U(2)} d u_{1} \int_{S U(2)} d u_{2} \\
& \left(\prod_{i=1}^{n+1} t_{m_{i} k_{i}}^{l_{i}}\left(u_{1}^{-1}\right) t_{\dot{m}_{i} \dot{k}_{i}}^{i_{i}}\left(u_{2}^{-1}\right)\right)\left(\prod_{j=n+2}^{n+m+1} t_{m_{j} k_{j}}^{l_{j}}\left(u_{1}\right) t_{\dot{m}_{j} \dot{k}_{j}}^{i_{j}}\left(u_{2}\right)\right) \times \\
& \int d^{4 m} x f_{k_{1}, \ldots, k_{n+m+1} ; \dot{k}_{1}, \ldots, \dot{k}_{n+m+1}}^{l_{1}, \ldots, l_{n+m+1} ; i_{1}, \ldots, i_{n+1}}\left(\left(x_{1}, x_{1}\right), \ldots,\left(x_{m}, x_{m}\right)\right) \times \\
& \left(\prod_{j=1}^{m} \theta\left(x_{j}^{0}\right)\left(\partial_{x_{j}}, \partial_{x_{j}}\right)^{q} t_{k_{n+1+j}, \dot{k}_{n+1+j}}^{l_{n+1+j}}\left(-\tilde{\partial}_{x_{j}}\right)\right) \phi\left(x_{1}, \ldots, x_{m}\right)
\end{aligned}
$$


Let us consider the case $m=n=1$. In view of the definition of the group $S U(2)$ we have $u^{-1}=u^{*}$. Hence the relations (2.6), (2.9), (2.43) imply the equality (2.35). Due to the relations (2.12), (2.13) any distribution (2.31) with the functions (2.35) satisfies the covariance relation (2.14).

Let us consider the case $m>2, n>1$. By making use of the relations (2.6), (2.7), (2.9) and the equality (2.43) we have the equality (2.36). Due to the relations (2.33), (2.34) any distribution (2.31) with the functions (2.36) satisfies the covariance relation (2.14). The theorem is proved.

If we insert the functions (2.35) into the series (2.31) for $m=n=1$, then this series will be finite due to the triangle condition for the Clebsch - Gordan coefficients. If we insert the functions (2.36) into the series (2.31) for $m>2, n>1$, then this series may be infinite. It is necessary to choose the functions $\phi\left(x_{1}, \ldots, x_{m}\right)$ for which the series is convergent. If $\phi\left(x_{1}, \ldots, x_{m}\right) \in D\left(\mathbf{R}^{4 m}\right)$, then the infinitely differentiable function $\phi\left(x_{1}, \ldots, x_{m}\right)$ has a compact support. For these functions we can formulate the the properties of spectrality and Lorentz covariance. We can not formulate the property of locality for these functions. If the Fourier transform

$$
\tilde{\phi}\left(p_{1}, \ldots, p_{m}\right)=\int d^{4 m} x \exp \left\{i \sum_{j=1}^{m}\left(p_{j}, x_{j}\right)\right\} \phi\left(x_{1}, \ldots, x_{m}\right)
$$

belongs to the space $D\left(\mathbf{R}^{4 m}\right)$, then the function (2.44) is infinitely differentiable and has a compact support. For these functions we can formulate the properties of locality and Lorentz covariance. We can not formulate the property of spectrality for these functions.

Due to $([9]$, Section 30)

$$
\begin{gathered}
(8 \pi)^{-1}\left(\partial_{x}, \partial_{x}\right)^{2}\left(\theta\left(x^{0}\right) \theta((x, x))\right)=\delta(x) \\
\left(\partial_{x}, \partial_{x}\right)\left(\theta\left(x^{0}\right) \theta((x, x))(x, x)^{n}\right)=4 n(n+1) \theta\left(x^{0}\right) \theta((x, x))(x, x)^{n-1}, n=1,2, \ldots
\end{gathered}
$$

Let us consider the distribution (2.31) for the case

$$
\begin{array}{r}
\left(\prod_{j=1}^{m}\left(\partial_{x_{j}}, \partial_{x_{j}}\right)^{q}\right)\left(\left(\prod_{j=1}^{m} \theta\left(x^{0}\right)\right) f_{m_{1}, \ldots, m_{n+m+1} ; \dot{m}_{1}, \ldots ., \dot{m}_{n+m+1}}^{l_{1}, \ldots, l_{n+m+1} ; \dot{l}_{1}, \ldots, i_{n+m+1}}\left(\left(x_{1}, x_{1}\right), \ldots,\left(x_{m}, x_{m}\right)\right)\right)= \\
a_{m_{1}, \ldots, m_{n+m+1} ; \dot{m}_{1}, \ldots, \dot{m}_{n+m+1}}^{l_{1}, \ldots, l_{n+m+1} ; \dot{l}_{1}, \ldots, i_{n+m+1}} \prod_{j=1}^{m} \delta\left(x_{j}\right) .
\end{array}
$$

The substitution of the equalities (2.45) into the series (2.31) yields

$$
\left.\sum_{k_{j}^{\mu} \in \mathbf{Z}_{+}, j=1, \ldots, m, \mu=0, \ldots, 3} a\left(k_{j}^{\mu}\right)\left(\prod_{j=1}^{m} \prod_{\mu=0}^{3}\left(\frac{\partial}{\partial x_{j}^{\mu}}\right)^{k_{j}^{\mu}}\right) \phi\left(x_{1}, \ldots, x_{m}\right)\right|_{x_{j}=0} .
$$

Lemma 2.3. If the series (2.46) is convergent for any function $\phi\left(x_{1}, \ldots, x_{m}\right) \in D\left(\mathbf{R}^{4 m}\right)$, then the number of the coefficients $a\left(k_{j}^{\mu}\right)$ which are not equal to zero is finite.

Proof. Let us prove that for any coefficients $b\left(k_{j}^{\mu}\right)$ there is the function $\phi\left(x_{1}, \ldots, x_{m}\right) \in$ $D\left(\mathbf{R}^{4 m}\right)$ such that

$$
\left.\left(\prod_{j=1}^{m} \prod_{\mu=0}^{3}\left(\frac{\partial}{\partial x_{j}^{\mu}}\right)^{k_{j}^{\mu}}\right) \phi\left(x_{1}, \ldots, x_{m}\right)\right|_{x_{j}=0}=b\left(k_{j}^{\mu}\right)
$$


It is sufficient to construct a function $\phi(x) \in D(\mathbf{R})$ satisfying one dimensional condition $(2.47)$

$$
\left.\left(\frac{d}{d x}\right)^{k} \phi(x)\right|_{x=0}=b(k) .
$$

Let a function $h(x) \in D(\mathbf{R})$ be equal to 1 in a neighborhood of the point $x=0$. We prove that the function

$$
\phi(x)=\sum_{k=0}^{\infty} a(k) \frac{x^{k}}{k !} h(x) \exp \left\{-|a(k)| x^{2}\right\}
$$

belongs to the space $D(\mathbf{R})$ for any numbers $a(k)$. It is easy to obtain the estimates

$$
\begin{gathered}
\left|\sum_{k=2}^{\infty} a(k) \frac{x^{k}}{k !} \exp \left\{-|a(k)| x^{2}\right\}\right| \leq\left(\sum_{k=2}^{\infty} \frac{|x|^{k-2}}{k !}\right) \sup _{k \in \mathbf{Z}_{+}, x \in \mathbf{R}}|a(k)| x^{2} \exp \left\{-|a(k)| x^{2}\right\}, \\
\sup _{k \in \mathbf{Z}_{+}, x \in \mathbf{R}}|a(k)| x^{2} \exp \left\{-|a(k)| x^{2}\right\} \leq \sup _{0 \leq x<\infty} x e^{-x}=e^{-1} .
\end{gathered}
$$

The estimates (2.50), (2.51) imply the absolute convergence of the series (2.49). If we differentiate every term of the series (2.49), the obtained series will be absolutely convergent. The proof is similar to the above one. The function (2.49) is infinitely differentiable and has a compact support. Hence $\phi(x) \in D(\mathbf{R})$.

The definition (2.49) implies

$$
\begin{array}{r}
\phi(0)=a(0), \\
\left.\left(\frac{d}{d x}\right)^{m} \phi(x)\right|_{x=0}=a(m)+\sum_{k=0}^{m-1} \sum_{n=0}^{\left[\frac{m}{2}\right]} C_{k n} a(k)|a(k)|^{n}
\end{array}
$$

where $C_{k n}$ is a combinatorial coefficient. We solve the recurrent relations (2.48), (2.52) and find the coefficients $a(k)$. The lemma is proved.

The Fourier transform of the distribution (2.46) is the power series. It is natural to define the series (2.46) for the functions $\phi\left(x_{1}, \ldots, x_{m}\right)$ whose Fourier transform (2.44) has a compact support.

\section{$3 \quad$ PCT, permutation invariance and spin condition}

We introduce the vacuum expectation values of the product of the quantum fields where the action of the permutation group is simple. Let us consider the vectors $p_{i j}=p_{j i} \in \mathbf{R}^{4}$, $1 \leq i<j \leq n+1, n=1,2, \ldots$ and the distribution

$$
f\left(p_{i j}, 1 \leq i<j \leq n+1\right) \in S^{\prime}\left(\mathbf{R}^{4 \frac{1}{2} n(n+1)}\right)
$$

with support in the product of the cones (1.7). The distribution

$$
\begin{array}{r}
\int d^{4 n} p * f\left(p_{1}, \ldots, p_{n}\right) \phi\left(p_{1}, \ldots, p_{n}\right)=\int\left(\prod_{1 \leq i<j \leq n+1} d^{4} p_{i j}\right) \\
f\left(p_{i j}, 1 \leq i<j \leq n+1\right) \phi\left(\sum_{1<j \leq n+1} p_{1 j}, \ldots, \sum_{1 \leq i \leq k, k<j \leq n+1} p_{i j}, \ldots, \sum_{1 \leq i \leq n} p_{i, n+1}\right)
\end{array}
$$


is called the multi - convolution of the distribution $f\left(p_{i j}, 1 \leq i<j \leq n+1\right)$.

Let us consider the complex vectors $z_{j} \in \mathbf{C}^{4}, j=1, \ldots, n+1$,

$$
\begin{gathered}
\operatorname{Im}\left(z_{j+1}-z_{j}\right) \in V_{+}, j=1, \ldots, n, \\
V_{+}=\left\{x \in \mathbf{R}^{4}: x^{0}>0,(x, x)>0\right\} .
\end{gathered}
$$

Hence

$$
\operatorname{Im}\left(z_{j}-z_{i}\right) \in V_{+}, 1 \leq i<j \leq n+1
$$

It is easy to verify

$$
\sum_{k=1}^{n}\left(\sum_{1 \leq i \leq k, k<j \leq n+1} p_{i j}, z_{k+1}-z_{k}\right)=\sum_{1 \leq i<j \leq n+1}\left(p_{i j}, z_{j}-z_{i}\right) .
$$

By making use of the relation (3.2) we can calculate the Fourier - Laplace transform of the multi - convolution (3.1)

$$
\begin{array}{r}
\int d^{4 n} p * f\left(p_{1}, \ldots, p_{n}\right) \exp \left\{\sqrt{-1} \sum_{k=1}^{n}\left(p_{k}, z_{k+1}-z_{k}\right)\right\}= \\
\int\left(\prod_{1 \leq i<j \leq n+1} d^{4} p_{i j}\right) f\left(p_{i j}, 1 \leq i<j \leq n+1\right) \exp \left\{\sqrt{-1} \sum_{1 \leq i<j \leq n+1}\left(p_{i j}, z_{j}-z_{i}\right)\right\} .
\end{array}
$$

The step function

$$
\theta(k)=\left\{\begin{array}{cc}
1, & k=0,1,2,3, \ldots \\
0, & k=-1,-2, \ldots
\end{array}\right.
$$

is the restriction of the step function (2.17) on the integers. Let $\pi$ be a permutation of the natural numbers $1, \ldots, n+1$. By using the changes $i \rightarrow \pi(i), j \rightarrow \pi(j)$ of the summation variables we have

$$
\begin{array}{r}
\sum_{1 \leq i<j \leq n+1}\left(p_{i j}, z_{j}-z_{i}\right)=\frac{1}{2} \sum_{i, j=1, \ldots, n+1, i \neq j}\left(p_{i j},(-1)^{\theta(i-j)}\left(z_{j}-z_{i}\right)\right)= \\
\sum_{1 \leq i<j \leq n+1}\left(p_{\pi(i) \pi(j)},(-1)^{\theta(\pi(i)-\pi(j))}\left(z_{\pi(j)}-z_{\pi(i)}\right)\right) .
\end{array}
$$

By making use of the relation (3.5) and the change $p_{\pi(i) \pi(j)} \rightarrow p_{i j}$ of the integration variables we get

$$
\begin{array}{r}
\int\left(\prod_{1 \leq i<j \leq n+1} d^{4} p_{i j}\right) f\left(p_{\pi(i) \pi(j)}, 1 \leq i<j \leq n+1\right) \times \\
\exp \left\{\sqrt{-1} \sum_{1 \leq i<j \leq n+1}\left(p_{i j}, z_{j}-z_{i}\right)\right\}= \\
\int\left(\prod_{1 \leq i<j \leq n+1} d^{4} p_{i j}\right) f\left(p_{i j}, 1 \leq i<j \leq n+1\right) \times \\
\exp \left\{\sqrt{-1} \sum_{1 \leq i<j \leq n+1}\left(p_{i j},(-1)^{\theta(\pi(i)-\pi(j))}\left(z_{\pi(j)}-z_{\pi(i)}\right)\right)\right\} .
\end{array}
$$


The right - hand side of the equality (3.6) and the action of the permutation $\pi$ on the function (3.3) of the variables $z_{1}, \ldots, z_{n+1}$ differ from each other in the sign multipliers $(-1)^{\theta(\pi(i)-\pi(j))}$ only.

Let us consider the distributions (1.4) for $n=1$. The distribution (1.6) has the form (2.31), (2.35) for $m=n=1$. Let the half - integers $l_{2}=\dot{l}_{1}, \dot{l}_{2}=l_{1}, l_{3}=l_{12}, m_{3}=m_{12}, \dot{m}_{3}=$ $\dot{m}_{12}$. This distribution is the vacuum expectation value of the product of the quantum field operator and its adjoint operator. The Clebsch - Gordan coefficient $C\left(l_{1}, i_{1}, l_{12} ; m_{1}, m_{2}, m_{12}\right)$ is not zero only in the case when the half - integer $l_{12}$ is equal to one of the half - integers $\left|l_{1}-\dot{l}_{1}\right|,\left|l_{1}-\dot{l}_{1}\right|+1, \ldots, l_{1}+\dot{l}_{1}-1, l_{1}+\dot{l}_{1}$. Hence

$$
2 l_{12}=2 l_{1}+2 \dot{l}_{1}=\left(2 l_{1}+2 \dot{l}_{1}\right)^{2}=\left(2 l_{1}+2 \dot{l}_{1}\right)\left(2 l_{2}+2 \dot{l}_{2}\right) \bmod 2 .
$$

\section{Spin condition}

We consider the infinite number of the quantum fields. Let the set of the natural numbers $1, \ldots, n+1$ be divided into the sets $A_{1}, \ldots, A_{N}$. Some of the sets $A_{1}, \ldots, A_{N}$ may be empty. With any natural number $i=1, \ldots, n+1$ there correspond the half-integers $l_{i}, \dot{l}_{i} \in 1 / 2 \mathbf{Z}_{+}$; $m_{i}=-l_{i},-l_{i}+1, \ldots, l_{i}-1, l_{i} ; \dot{m}_{i}=-\dot{l}_{i},-\dot{l}_{i}+1, \ldots, \dot{l}_{i}-1, \dot{l}_{i}$ such that $l_{i}=l_{j}, \dot{l}_{i}=\dot{l}_{j}$ if the natural numbers $i, j$ belong to the same set $A_{k}$.

The vacuum expectation value of the product of $n+1, n=1,2, \ldots$, quantum fields is the distribution

$$
\begin{array}{r}
W_{m_{1}, \ldots, m_{n+1} ; \dot{m}_{1}, \ldots, \dot{m}_{n+1}}^{l_{1}, \ldots, l_{n+1} ; i_{1}, \ldots, i_{n+1}}\left(A_{1}, \ldots, A_{N} ; x_{2}-x_{1}, \ldots, x_{n+1}-x_{n}\right)= \\
\int\left(\prod_{1 \leq i<j \leq n+1} d^{4} p_{i j}\right) F_{m_{1}, \ldots, m_{n+1} ; \dot{m}_{1}, \ldots, \dot{m}_{n+1}}^{l_{1}, \ldots, l_{n+1} ; i_{1}, \ldots, i_{n+1}}\left(A_{1}, \ldots, A_{N} ; p_{i j}, 1 \leq i<j \leq n+1\right) \times \\
\exp \left\{\sqrt{-1} \sum_{k=1}^{n}\left(\sum_{1 \leq i \leq k, k<j \leq n+1} p_{i j}, x_{k+1}-x_{k}\right)\right\} .
\end{array}
$$

The distribution

$$
\begin{aligned}
& \int\left(\prod_{1 \leq i<j \leq n+1} d^{4} p_{i j}\right) F_{m_{1}, \ldots, m_{n+1} ; \dot{m}_{1}, \ldots, \dot{m}_{n+1}}^{l_{1}, \ldots, l_{n+1} ; i_{1}, \ldots, i_{n+1}}\left(A_{1}, \ldots, A_{N} ; p_{i j}, 1 \leq i<j \leq n+1\right) \times \\
& \phi\left(p_{i j}, 1 \leq i<j \leq n+1\right)= \\
& \sum_{l_{i j} \in 1 / 2 \mathbf{Z}_{+}, 1 \leq i<j \leq n+1} \sum_{m_{i j}, \dot{m}_{i j}=-l_{i j},-l_{i j}+1, \ldots, l_{i j}-1, l_{i j}, 1 \leq i<j \leq n+1} \int\left(\prod_{1 \leq i<j \leq n+1} d^{4} p_{i j}\right) \\
& f_{m_{i}, \dot{m}_{i}, 1 \leq i \leq n+1 ; m_{i j}, \dot{m}_{i j}, 1 \leq i<j \leq n+1}^{l_{i}, i_{i}, 1 \leq i \leq n+1 ; l_{i j} 1 \leq i<j \leq n+1}\left(A_{1}, \ldots, A_{N} ;\left(p_{i j}, p_{i j}\right), 1 \leq i<j \leq n+1\right) \times \\
& \left(\prod_{1 \leq i<j \leq n+1} \theta\left(p_{i j}^{0}\right)\left(\partial_{p_{i j}}, \partial_{p_{i j}}\right)^{q} t_{m_{i j} \dot{m}_{i j}}^{l_{i j}}\left(-\tilde{\partial}_{p_{i j}}\right)\right) \phi\left(p_{i j}, 1 \leq i<j \leq n+1\right)
\end{aligned}
$$

is defined on any test function $\phi\left(p_{i j}, 1 \leq i<j \leq n+1\right)$ whose Fourier transform is an infinitely differentiable function with a compact support. $q$ is a natural number. $2 \times 2$ matrix $\tilde{\partial}_{x}$ is given by the relation (2.19). The polynomial $t_{m n}^{l}(A)$ is given by the relation (2.4). The continuous function

$$
f_{m_{i}, \dot{m}_{i}, 1 \leq i \leq n+1 ; m_{i j}, \dot{m}_{i j}, 1 \leq i<j \leq n+1}^{l_{i}, \dot{l}_{i}, 1 \leq i \leq n+1 ; l_{i j} 1 \leq i<j \leq n+1}\left(A_{1}, \ldots, A_{N} ; s_{i j}, 1 \leq i<j \leq n+1\right)
$$


with a support in the product of the positive semi - axes is polynomial bounded. The parity of any integer $2 l_{i j}$ in the equality (3.9) is given by the relation

$$
2 l_{i j}=\left(2 l_{i}+2 \dot{l}_{i}\right)\left(2 l_{j}+2 \dot{l}_{j}\right) \bmod 2,1 \leq i<j \leq n+1
$$

Any term in the series (3.9) is a tempered distribution from the space $S^{\prime}\left(\mathbf{R}^{4 \frac{1}{2} n(n+1)}\right)$. This distribution has a support in the product of the closed upper light cones (1.7). The support of the infinite series (3.9) is not defined.

Theorem 3.1. Let for any permutation $\pi$ of the natural numbers $1, \ldots, n+1$ the distribution (3.9) satisfy the relation

$$
\begin{array}{r}
F_{m_{\pi(i)}, \dot{m}_{\pi(i)}, 1 \leq i \leq n+1}^{l_{\pi(i)}, i_{\pi(i)}, 1 \leq i \leq n+1}\left(\pi\left(A_{i}\right), 1 \leq i \leq N ; p_{\pi(i) \pi(j)}, 1 \leq i<j \leq n+1\right)= \\
F_{m_{1}, \ldots, m_{n+1} ; \dot{m}_{1}, \ldots, \dot{m}_{n+1}}^{l_{1}, \ldots, l_{n+1} ; i_{1}, \ldots, i_{n+1}}\left(A_{1}, \ldots, A_{N} ; p_{i j}, 1 \leq i<j \leq n+1\right)
\end{array}
$$

Then for the vectors $x_{i} \in \mathbf{R}^{4}, i=1, \ldots, n+1,\left(x_{j}-x_{i}, x_{j}-x_{i}\right)<0,1 \leq i<j \leq n+1$, and for any permutation $\pi$ of the natural numbers $1, \ldots, n+1$ the distribution (3.8) satisfies the relation

$$
\begin{array}{r}
(-1)^{\sigma_{l}(\pi)} W_{m_{\pi(i)}, \dot{m}_{\pi(i)}, 1 \leq i \leq n+1}^{l_{\pi(i)}, i_{\pi(i)}, 1 \leq i \leq n+1}\left(\pi\left(A_{i}\right), 1 \leq i \leq N ; x_{\pi(i+1)}-x_{\pi(i)}, 1 \leq i \leq n\right)= \\
W_{m_{1}, \ldots, m_{n+1} ; \dot{m}_{1}, \ldots, \dot{m}_{n+1}}^{l_{1}, \ldots, l_{n+1} ; i_{1}, \ldots, i_{n+1}}\left(A_{1}, \ldots, A_{N} ; x_{2}-x_{1}, \ldots, x_{n+1}-x_{n}\right)
\end{array}
$$

where the number

$$
\sigma_{l}(\pi)=\sum_{1 \leq i<j \leq n+1}\left(2 l_{i}+2 \dot{l}_{i}\right)\left(2 l_{j}+2 \dot{l}_{j}\right) \theta(\pi(i)-\pi(j)) \bmod 2 .
$$

Proof. Let a function $\phi\left(x_{1}, \ldots, x_{n+1}\right) \in D\left(\mathbf{R}^{4(n+1)}\right)$. Due to the definitions (3.8), (3.9) and the relation $(3.2)$

$$
\begin{aligned}
& \int d^{4(n+1)} x W_{m_{1}, \ldots, m_{n+1} ; \dot{m}_{1}, \ldots, m_{n+1}}^{l_{1}, \ldots, l_{n+1} i_{1}, \ldots, i_{n+1}}\left(A_{1}, \ldots, A_{N} ; x_{2}-x_{1}, \ldots, x_{n+1}-x_{n}\right) \phi\left(x_{1}, \ldots, x_{n+1}\right)= \\
& \sum_{l_{i j} \in 1 / 2 \mathbf{Z}_{+}, 1 \leq i<j \leq n+1} \sum_{m_{i j}, \dot{m}_{i j}=-l_{i j},-l_{i j}+1, \ldots, l_{i j}-1, l_{i j}, 1 \leq i<j \leq n+1} \int\left(\prod_{1 \leq i<j \leq n+1} d^{4} p_{i j}\right) \\
& f_{m_{i}, \dot{m}_{i}, 1 \leq i \leq n+1 ; m_{i j}, \dot{m}_{i j}, 1 \leq i<j \leq n+1}^{l_{i}, i_{i}, 1 \leq i \leq n+1 ; l_{i j} 1 \leq i<j \leq n+1}\left(A_{1}, \ldots, A_{N} ;\left(p_{i j}, p_{i j}\right), 1 \leq i<j \leq n+1\right) \times \\
& \left(\prod_{1 \leq i<j \leq n+1} \theta\left(p_{i j}^{0}\right)\left(\partial_{p_{i j}}, \partial_{p_{i j}}\right)^{q} t_{m_{i j} \dot{m}_{i j}}^{l_{i j}}\left(-\tilde{\partial}_{p_{i j}}\right)\right) \times \\
& \int d^{4(n+1)} x \phi\left(x_{1}, \ldots, x_{n+1}\right) \exp \left\{\sqrt{-1} \sum_{1 \leq i<j \leq n+1}\left(p_{i j}, x_{j}-x_{i}\right)\right\} \text {. }
\end{aligned}
$$

In view of ([9], Section 26.3) we have

$$
\begin{array}{r}
\int\left(\prod_{1 \leq i<j \leq n+1} d^{4} p_{i j}\right) \\
f_{m_{i}, \dot{m}_{i}, 1 \leq i \leq n+1 ; m_{i j}, \dot{m}_{i j}, 1 \leq i<j \leq n+1}^{l_{i}, i_{i}, 1 \leq i \leq n+1 ; l_{i j} 1 \leq i<j \leq n+1}\left(A_{1}, \ldots, A_{N} ;\left(p_{i j}, p_{i j}\right), 1 \leq i<j \leq n+1\right) \times
\end{array}
$$




$$
\begin{aligned}
& \left(\prod_{1 \leq i<j \leq n+1} \theta\left(p_{i j}^{0}\right)\left(\partial_{p_{i j}}, \partial_{p_{i j}}\right)^{q} t_{m_{i j} \dot{m}_{i j}}^{l_{i j}}\left(-\tilde{\partial}_{p_{i j}}\right)\right) \times \\
& \int d^{4(n+1)} x \phi\left(x_{1}, \ldots, x_{n+1}\right) \exp \left\{\sqrt{-1} \sum_{1 \leq i<j \leq n+1}\left(p_{i j}, x_{j}-x_{i}\right)\right\}= \\
& \lim _{z_{i} \rightarrow 0, \operatorname{Im}\left(z_{j}-z_{i}\right) \in V_{+}, 1 \leq i<j \leq n+1} \int\left(\prod_{1 \leq i<j \leq n+1} d^{4} p_{i j}\right) \\
& f_{m_{i}, \dot{m}_{i}, 1 \leq i \leq n+1 ; m_{i j}, \dot{m}_{i j}, 1 \leq i<j \leq n+1}^{l_{i}, i_{i}, 1 \leq i \leq n+1 ; l_{i j} 1 \leq i<j \leq n+1}\left(A_{1}, \ldots, A_{N} ;\left(p_{i j}, p_{i j}\right), 1 \leq i<j \leq n+1\right) \times \\
& \left(\prod_{1 \leq i<j \leq n+1} \theta\left(p_{i j}^{0}\right)\left(\partial_{p_{i j}}, \partial_{p_{i j}}\right)^{q} t_{m_{i j} \dot{m}_{i j}}^{l_{i j}}\left(-\tilde{\partial}_{p_{i j}}\right)\right) \times \\
& \int d^{4(n+1)} \operatorname{Re} z \phi\left(\operatorname{Re} z_{1}, \ldots, \operatorname{Re} z_{n+1}\right) \exp \left\{\sqrt{-1} \sum_{1 \leq i<j \leq n+1}\left(p_{i j}, z_{j}-z_{i}\right)\right\} .
\end{aligned}
$$

Let a function $f(s)$ with a support in the positive semi - axis be polynomial bounded. We can represent any vector $y \in V_{+}$in the form

$$
\begin{gathered}
\tilde{y}=(y, y)^{1 / 2} g(t, z) g(t, z)^{*}, \\
g(t, z)=\left(\begin{array}{cc}
t^{-1} & 0 \\
z & t
\end{array}\right)
\end{gathered}
$$

where $t$ is a positive number and $z$ is a complex number.

By making the change of the variables

$$
\tilde{p}=\mu^{1 / 2} g(t, z) u(\phi, \theta, \psi)\left((\cosh s) \sigma^{0}+(\sinh s) \sigma^{3}\right) u(\phi, \theta, \psi)^{*} g(t, z)^{*}
$$

where $2 \times 2$ - matrix $u(\phi, \theta, \psi)$ is given by the relation $(2.41)$ we have

$$
\begin{array}{r}
\int d^{4} p \theta\left(p^{0}\right) f((p, p)) \exp \{-(p, y)\}= \\
2 \pi \int_{0}^{\infty} d \mu \int_{0}^{\infty} d s \int_{S U(2)} d u(\phi, \theta, \psi) \mu f(\mu) \sinh ^{2} s \exp \left\{-\mu^{1 / 2}(y, y)^{1 / 2} \cosh s\right\}
\end{array}
$$

The normalized Haar measure $d u(\phi, \theta, \psi)$ on the group $S U(2)$ is given by the relation (2.42). By making use of the relation ([10], relation $7.12(21))$

$$
K_{\nu}(z)=\int_{0}^{\infty} d s(\cosh \nu s) \exp \{-z \cosh s\}, \operatorname{Re} z>0
$$

and the relation $([10]$, relation $7.11(25))$

$$
K_{\nu+1}(z)-K_{\nu-1}=2 \nu z^{-1} K_{\nu}(z)
$$

we can rewrite the equality (3.18) in the form

$$
\int d^{4} p \theta\left(p^{0}\right) f((p, p)) \exp \{-(p, y)\}=2 \pi \int_{0}^{\infty} d \mu \mu f(\mu)(\mu(y, y))^{-1 / 2} K_{1}\left((\mu(y, y))^{1 / 2}\right) .
$$


Due to $([10]$, relation $7.2 .5(37))$

$$
\begin{array}{r}
\mu(\mu(y, y))^{-1 / 2} K_{1}\left((\mu(y, y))^{1 / 2}\right)= \\
\frac{1}{2} \mu \ln \left(\frac{1}{2}(\mu(y . y))^{1 / 2}\right) \sum_{m=0}^{\infty} \frac{1}{m !(m+1) !}\left(\frac{1}{4} \mu(y, y)\right)^{m}+ \\
(y, y)^{-1}-2^{-3 / 2} \mu \sum_{m=0}^{\infty}\left(\frac{1}{4} \mu(y, y)\right)^{m} \frac{\psi(m+2)+\psi(m+1)}{m !(m+1) !}
\end{array}
$$

where the logarithmic derivative of the gamma - function

$$
\psi(z)=\frac{d}{d z}(\ln \Gamma(z))
$$

For $-\frac{3 \pi}{2}<\arg z<\frac{3 \pi}{2}$ the asymptotic relation $([10]$, relation $7.13(7))$ is valid

$$
K_{\nu}(z)=\left(\frac{\pi}{2 z}\right)^{1 / 2} e^{-z}\left(1+O\left(|z|^{-1}\right)\right)
$$

In view of the relations (3.22), (3.23) both parts of the equality (3.21) as the functions of the variable $i y$ have an analytic continuation into the tube domain $\mathbf{R}^{4}+i V_{+}$

$$
\int d^{4} p \theta\left(p^{0}\right) f((p, p)) \exp \{i(p, z)\}=2 \pi \int_{0}^{\infty} d \mu \mu f(\mu)(-\mu(z, z))^{-1 / 2} K_{1}\left((-\mu(z, z))^{1 / 2}\right)
$$

where a complex vector $z=x+i y \in \mathbf{R}^{4}+i V_{+}$.

Let the compact support of the function $\phi\left(x_{1}, \ldots, x_{n+1}\right)$ lie in the domain $\left(x_{j}-x_{i}, x_{j}-x_{i}\right)<$ $0,1 \leq i<j \leq n+1$. The relations (3.16), (3.22) - (3.24) imply

$$
\begin{aligned}
& \int\left(\prod_{1 \leq i<j \leq n+1} d^{4} p_{i j}\right) \\
& f_{m_{i}, \dot{m}_{i}, 1 \leq i \leq n+1 ; m_{i j}, m_{i j}, 1 \leq i<j \leq n+1}^{l_{i}, i_{i}, 1 \leq i \leq n+1 ; l_{i j} 1 \leq i<j \leq n+1}\left(A_{1}, \ldots, A_{N} ;\left(p_{i j}, p_{i j}\right), 1 \leq i<j \leq n+1\right) \times \\
& \left(\prod_{1 \leq i<j \leq n+1} \theta\left(p_{i j}^{0}\right)\left(\partial_{p_{i j}}, \partial_{p_{i j}}\right)^{q} t_{m_{i j} \dot{m}_{i j}}^{l_{i j}}\left(-\tilde{\partial}_{p_{i j}}\right)\right) \times \\
& \int d^{4(n+1)} x \phi\left(x_{1}, \ldots, x_{n+1}\right) \exp \left\{\sqrt{-1} \sum_{1 \leq i<j \leq n+1}\left(p_{i j}, x_{j}-x_{i}\right)\right\}= \\
& \int d^{4(n+1)} x \phi\left(x_{1}, \ldots, x_{n+1}\right) \times \\
& \left(\prod_{1 \leq i<j \leq n+1}\left(2 \pi\left(-\left(x_{j}-x_{i}, x_{j}-x_{i}\right)\right)^{q} t_{m_{i j} \dot{m}_{i j}}^{l_{i j}}\left(\sqrt{-1}\left(\tilde{x}_{i}-\tilde{x}_{j}\right)\right)\right)\right) \times \\
& \left(\prod_{1 \leq i<j \leq n+1} \int_{0}^{\infty} \mu_{i j} d \mu_{i j}\right) \\
& f_{m_{i}, \dot{m}_{i}, 1 \leq i \leq n+1 ; m_{i j}, \dot{m}_{i j}, 1 \leq i<j \leq n+1}^{l_{i}, \dot{l}_{i}, 1 \leq i \leq n+1 ; l_{i j} 1 \leq i<j \leq n+1}\left(A_{1}, \ldots, A_{N} ; \mu_{i j}, 1 \leq i<j \leq n+1\right) \times \\
& \left(\prod_{1 \leq i<j \leq n+1}\left(-\mu_{i j}\left(x_{j}-x_{i}, x_{j}-x_{i}\right)\right)^{-1 / 2} K_{1}\left(\left(-\mu_{i j}\left(x_{j}-x_{i}, x_{j}-x_{i}\right)\right)^{1 / 2}\right)\right)
\end{aligned}
$$


We insert the left - hand side of the equality (3.12) into the right - hand side of the equality (3.8). In view of the relations (3.6), (3.25) the obtained distribution coincides with the left hand side of the equality (3.13) for $\left(x_{j}-x_{i}, x_{j}-x_{i}\right)<0,1 \leq i<j \leq n+1$. (The polynomial (2.4) is homogeneous of the degree $2 l$. The parity of the integer $2 l_{i j}$ is given by the relation (3.11).) The theorem is proved.

The quantum field of the number $i$ is called anti - commuting if

$$
2 l_{i}+2 \dot{l}_{i}=1 \bmod 2 .
$$

The definition (3.26) is consistent with the relation (3.7). The set of numbers corresponding to the anti - commuting quantum fields is denoted by $F$. The definitions (3.14), (3.26) imply

$$
\sigma_{l}(\pi)=\sum_{i, j \in F, i \neq j} \theta(\pi(i)-\pi(j)) \theta(j-i) \bmod 2 .
$$

According to the relation (3.27) the multiplier $(-1)^{\sigma_{l}(\pi)}$ in the relation (3.13) coincides with the multiplier $(-1)^{M}$ in the relation $(1.12)$.

Lemma 3.2. Let the permutation $\lambda$ of the natural numbers $1, \ldots, n+1$ transform the set $F$ into itself. Then for any permutation $\pi$ of the natural numbers $1, \ldots, n+1$ the following relation is valid

$$
\sigma_{l}(\pi \circ \lambda)=\sigma_{l}(\pi)+\sigma_{l}(\lambda) \bmod 2 .
$$

Proof. For any natural numbers $i, j \in F, i \neq j$, the definition (3.4) implies

$$
\theta(\lambda(i)-\lambda(j))+\theta(\lambda(j)-\lambda(i))=1
$$

By using this relation and the relation (3.27) we get

$$
\begin{array}{r}
\sigma_{l}(\pi \circ \lambda)=\sum_{i, j \in F, i \neq j} \theta(\pi(\lambda(i))-\pi(\lambda(j))) \theta(\lambda(j)-\lambda(i)) \theta(j-i)+ \\
\sum_{i, j \in F, i \neq j} \theta(\pi(\lambda(i))-\pi(\lambda(j))) \theta(\lambda(i)-\lambda(j)) \theta(j-i) \bmod 2 .
\end{array}
$$

For any natural numbers $i, j \in F, i \neq j$,

$$
\theta(j-i)=1+\theta(i-j) \bmod 2
$$

The substitution of this relation into the right - hand side of the equality (3.29) yields

$$
\begin{array}{r}
\sigma_{l}(\pi \circ \lambda)=\sum_{i, j \in F, i \neq j} \theta(\pi(\lambda(i))-\pi(\lambda(j))) \theta(\lambda(j)-\lambda(i))+ \\
\sum_{i, j \in F, i \neq j} \theta(\pi(\lambda(i))-\pi(\lambda(j))) \theta(\lambda(j)-\lambda(i)) \theta(i-j)+ \\
\sum_{i, j \in F, i \neq j} \theta(\pi(\lambda(i))-\pi(\lambda(j))) \theta(\lambda(i)-\lambda(j)) \theta(j-i) \bmod 2 .
\end{array}
$$

The permutation $\lambda$ transforms the set $F$ into itself. Let $\lambda^{-1}$ be the inverse permutation. We change the summation variables $i \rightarrow \lambda^{-1}(i), j \rightarrow \lambda^{-1}(j)$ in the first sum in the right - hand side of the equality (3.30) and $i \rightarrow j, j \rightarrow i$ in the second sum. Now the relation (3.30) and the relation

$$
\theta(\pi(\lambda(i))-\pi(\lambda(j)))+\theta(\pi(\lambda(j))-\pi(\lambda(i)))=1
$$


imply the equality (3.28). The lemma is proved.

For the permutation $\tau(i)=n+2-i, i=1, \ldots, n+1$, we have

$$
\begin{gathered}
\sum_{1 \leq i<j \leq n+1}\left(p_{i j},(-1)^{\theta(\tau(i)-\tau(j))}\left(z_{\tau(j)}-z_{\tau(i)}\right)\right)= \\
\sum_{k=1}^{n}\left(\sum_{1 \leq i \leq k, k<j \leq n+1} p_{i j}, z_{n+2-k}-z_{n+1-k}\right) .
\end{gathered}
$$

Suppose that the series (3.9) are finite. Then the equalities (3.8), (3.31) and the relations (3.6), (3.12) for the permutation $\tau$ imply

$$
\begin{array}{r}
W_{m_{1}, \ldots, m_{n+1} ; \dot{m}_{1}, \ldots, \dot{m}_{n+1}}^{l_{1}, \ldots, l_{n+1} ; i_{1}, i_{n+1}}\left(A_{1}, \ldots, A_{N} ; x_{2}-x_{1}, \ldots, x_{n+1}-x_{n}\right)= \\
W_{m_{n+1}, \ldots, m_{1} ; \dot{m}_{n+1}, \ldots, \dot{m}_{1}}^{l_{n+1}, \ldots, l_{1} ; i_{n+1}, \ldots, \dot{l}_{1}}\left(\tau\left(A_{1}\right), \ldots, \tau\left(A_{N}\right) ; x_{n+1}-x_{n}, x_{n}-x_{n-1}, \ldots, x_{2}-x_{1}\right) .
\end{array}
$$

In order to obtain the equality (3.32) we use also the fact that the distribution (3.8) is a boundary value of the Fourier - Laplace transform of the multi - convolution (3.1) of the distribution (3.9) ([9], Section 26.3).

Due to $([2]$, relation $(4-30))$ the vacuum expectation value of the product of $n+1$ quantum fields is the distribution

$$
W_{\mu \cdots \nu}\left(\xi_{1}, \ldots, \xi_{n}\right)=\left(\Psi_{0}, \psi_{\mu}\left(x_{1}\right) \cdots \psi_{\nu}\left(x_{n+1}\right) \Psi_{0}\right)
$$

where $\xi_{j}=x_{j}-x_{j+1}, j=1, \ldots, n$. In view of ([2], Theorem $\left.4-7\right)$ the condition of the invariance under PCT transformation is

$$
\left(\Psi_{0}, \psi_{\mu}\left(x_{1}\right) \cdots \psi_{\nu}\left(x_{n+1}\right) \Psi_{0}\right)=i^{F}(-1)^{J}\left(\Psi_{0}, \psi_{\nu}\left(-x_{n+1}\right) \cdots \psi_{\mu}\left(-x_{1}\right) \Psi_{0}\right)
$$

The multipliers $(-1)^{J}$ and $i^{F}$ are defined by the following relations $([2]$, relations $(4-32)$ and $(4-36))$

$$
\begin{gathered}
W_{\mu \cdots \nu}\left(\xi_{1}, \ldots, \xi_{n}\right)=(-1)^{J} W_{\mu \cdots \nu}\left(-\xi_{1}, \ldots,-\xi_{n}\right) \\
\left(\Psi_{0}, \psi_{\mu}\left(x_{1}\right) \cdots \psi_{\nu}\left(x_{n+1}\right) \Psi_{0}\right)=i^{F}\left(\Psi_{0}, \psi_{\nu}\left(x_{n+1}\right) \cdots \psi_{\mu}\left(x_{1}\right) \Psi_{0}\right) .
\end{gathered}
$$

The function (3.33) is holomorphic at the point $x_{1}, \ldots, x_{n+1}$.

Let us prove that for the finite series (3.9) the distribution (3.8), (3.9) satisfies the relations (3.35), (3.36) and the multiplier $i^{F}(-1)^{J}=1$. Therefore PCT invariance corresponds with the relation (3.12) for the permutation $\tau(i)=n+2-i, i=1, \ldots, n+1$.

In view of the equalities (3.23), (3.25) for the finite series (3.9) the function (3.8), (3.9) is holomorphic at the point $\left(x_{j}-x_{i}, x_{j}-x_{i}\right)<0,1 \leq i<j \leq n+1$. Then the relation (3.13) for the permutation $\tau$ corresponds to the relation (3.36). The multiplier

$$
i^{F}=(-1)^{\sigma_{l}(\tau)}=(-1)^{\sum_{1 \leq i<j \leq n+1}\left(2 l_{i}+2 i_{i}\right)\left(2 l_{j}+2 i_{j}\right)}
$$

The equalities (3.11), (3.25) imply

$$
\begin{array}{r}
W_{m_{1}, \ldots, m_{n+1} ; \dot{m}_{1}, \ldots, \dot{m}_{n+1}}^{l_{1}, \ldots, l_{n+1} ; i_{1}, i_{n+1}}\left(A_{1}, \ldots, A_{N} ; x_{2}-x_{1}, \ldots, x_{n+1}-x_{n}\right)= \\
(-1)^{J} W_{m_{1}, \ldots, m_{n+1} ; m_{1}, \ldots, \dot{m}_{n+1}}^{l_{1}, \ldots, l_{n+1} ; i_{1}, \ldots, i_{n+1}}\left(A_{1}, \ldots, A_{N} ;-\left(x_{2}-x_{1}\right), \ldots,-\left(x_{n+1}-x_{n}\right)\right) \\
(-1)^{J}=(-1)^{\sum_{1 \leq i<j \leq n+1}\left(2 l_{i}+2 i_{i}\right)\left(2 l_{j}+2 i_{j}\right)}
\end{array}
$$


The relation (3.38) corresponds to the relation (3.35). The multipliers (3.37), (3.39) satisfy the relation $i^{F}(-1)^{J}=1$.

\section{Permutation invariance}

The vacuum expectation value of the product of $n+1, n=1,2, \ldots$, quantum fields is the distribution (3.8), (3.9) where the functions (3.10) satisfy the relation

$$
\begin{array}{r}
f_{m_{\pi(i)}, \dot{m}_{\pi(i)}, 1 \leq i \leq n+1 ; m_{\pi(i) \pi(j)}, \dot{m}_{\pi(i) \pi(j)}, 1 \leq i<j \leq n+1}^{l_{\pi(i)}, i_{i(i)}, 1 \leq i \leq n+1 ; l_{\pi(i) \pi(j)} 1 \leq i<j \leq n+1} \\
\left(\pi\left(A_{i}\right), 1 \leq i \leq N ; s_{\pi(i) \pi(j)}, 1 \leq i<j \leq n+1\right)= \\
\left.f_{m_{i}, \dot{m}_{i}, 1 \leq i \leq n+1 ; m_{i j}, \dot{m}_{i j}, 1 \leq i<j \leq n+1}^{l_{i}, i_{i}, 1 \leq i \leq n+1 ; l_{i j} 1 \leq i<j \leq n+1}, 1 \leq i \leq N ; s_{i j}, 1 \leq i<j \leq n+1\right)
\end{array}
$$

for any permutation $\pi$ of the natural numbers $1, \ldots, n+1$.

Lemma 3.3. The equality (3.40) implies the equality (3.12).

Proof. Since $p_{j i}=p_{i j}, l_{j i}=l_{i j}, m_{j i}=m_{i j}, \dot{m}_{j i}=\dot{m}_{i j}$ we have for any permutation $\pi$ of the natural numbers $1, \ldots, n+1$

$$
\begin{array}{r}
\prod_{1 \leq i<j \leq n+1} \theta\left(p_{i j}^{0}\right)\left(\partial_{p_{i j}}, \partial_{p_{i j}}\right)^{q} t_{m_{i j} \dot{m}_{i j}}^{l_{i j}}\left(-\tilde{\partial}_{p_{i j}}\right)= \\
\prod_{1 \leq i<j \leq n+1} \theta\left(p_{\pi(i) \pi(j)}^{0}\right)\left(\partial_{p_{\pi(i) \pi(j)}}, \partial_{p_{\pi(i) \pi(j)}}\right)^{q} t_{m_{\pi(i) \pi(j)} \dot{m}_{\pi(i) \pi(j)}}^{l_{\pi(i) \pi(j)}}\left(-\tilde{\partial}_{p_{\pi(i) \pi(j)}}\right) .
\end{array}
$$

By making use of the relation (3.41) and the changes of the summation variables $l_{\pi(i) \pi(j)} \rightarrow l_{i j}$, $m_{\pi(i) \pi(j)} \rightarrow m_{i j}, \dot{m}_{\pi(i) \pi(j)} \rightarrow \dot{m}_{i j}$ we get

$$
\begin{aligned}
& \sum_{l_{i j} \in 1 / 2 \mathbf{Z}_{+}, 1 \leq i<j \leq n+1} \sum_{m_{i j}, \dot{m}_{i j}=-l_{i j},-l_{i j}+1, \ldots, l_{i j}-1, l_{i j}, 1 \leq i<j \leq n+1} \int\left(\prod_{1 \leq i<j \leq n+1} d^{4} p_{i j}\right) \\
& f_{\pi(i)}^{l_{\pi(i)}, l_{\pi(i)}, 1 \leq i \leq n+1 ; l_{\pi(i) \pi(j)} 1 \leq i<j \leq n+1} \\
& f_{m_{\pi(i)}, \dot{m}_{\pi(i)}, 1 \leq i \leq n+1 ; m_{\pi(i) \pi(j)}, \dot{m}_{\pi(i) \pi(j)}, 1 \leq i<j \leq n+1}^{l_{\pi(i)}, l_{\pi(i)}, 1 \leq i \leq n+1 ; l_{\pi(i) \pi(j)}} \\
& \left(\pi\left(A_{i}\right), 1 \leq i \leq N ;\left(p_{\pi(i) \pi(j)}, p_{\pi(i) \pi(j)}\right), 1 \leq i<j \leq n+1\right) \times \\
& \left(\prod_{1 \leq i<j \leq n+1} \theta\left(p_{i j}^{0}\right)\left(\partial_{p_{i j}}, \partial_{p_{i j}}\right)^{q} t_{m_{i j} \dot{m}_{i j}}^{l_{i j}}\left(-\tilde{\partial}_{p_{i j}}\right)\right) \phi\left(p_{i j}, 1 \leq i<j \leq n+1\right)= \\
& \int\left(\prod_{1 \leq i<j \leq n+1} d^{4} p_{i j}\right) \\
& F_{m_{\pi(i)}, \dot{m}_{\pi(i)}, 1 \leq i \leq n+1}^{l_{\pi(i)} i_{\pi(i \leq 1 \leq n+1}}\left(\pi\left(A_{i}\right), 1 \leq i \leq N ; p_{\pi(i) \pi(j)}, 1 \leq i<j \leq n+1\right) \times \\
& \phi\left(p_{i j}, 1 \leq i<j \leq n+1\right) .
\end{aligned}
$$

We insert the left - hand side of the equality (3.40) into the right - hand side of the equality (3.9) instead of the function (3.10). The obtained distribution coincides with the left - hand side of the equality (3.42). Hence the equalities (3.40), (3.42) imply the equality (3.12). The lemma is proved.

\section{Lorentz covariance}

The vacuum expectation value of the product of $n+1, n=1,2, \ldots$, quantum fields is the distribution (3.8), (3.9).

For $n=1$ the function (3.10) is

$$
\begin{array}{r}
f_{m_{1}, m_{2} ; \dot{m}_{1}, \dot{m}_{2} ; m_{12}, \dot{m}_{12}}^{l_{1}, l_{2} ; \dot{l}_{1}, \dot{l}_{2} ; l_{1}}\left(A_{1}, \ldots, A_{N} ; s_{12}\right)= \\
f^{l_{1}, l_{2} ; l_{1}, l_{2} ; l_{12}}\left(A_{1}, \ldots, A_{N} ; s_{12}\right) C\left(l_{1}, l_{2}, l_{12} ; m_{1}, m_{2}, m_{12}\right) C\left(\dot{l}_{1}, \dot{l}_{2}, l_{12} ; \dot{m}_{1}, \dot{m}_{2}, \dot{m}_{12}\right)
\end{array}
$$


where a continuous function $f^{l_{1}, l_{2} ; \dot{l}_{1}, i_{2} ; l_{12}}\left(A_{1}, \ldots, A_{N} ; s_{12}\right)$ with a support in the positive semi axis is polynomial bounded; the Clebsch - Gordan coefficient $C\left(l_{1}, l_{2}, l_{3} ; m_{1}, m_{2}, m_{3}\right)$ is given by the relation $(2.8)$.

For $n>1$ the function (3.10) is

$$
\begin{array}{r}
f_{m_{i}, \dot{m}_{i}, 1 \leq i \leq n+1 ; m_{i j}, \dot{m}_{i j}, 1 \leq i<j \leq n+1}^{l_{1} \dot{l}_{i}, 1 \leq i \leq n+1 ; l_{i} 1 \leq i \leq j+1} \sum_{j_{1}, \ldots, j_{\frac{1}{2} n(n+3)-2} ; j_{1}^{\prime}, \ldots, j_{\frac{1}{2} n(n+3)-2}^{\prime} \in 1 / 2 \mathbf{Z}_{+}}\left(A_{1}, \ldots, A_{N} ; s_{i j}, 1 \leq i<j \leq n+1\right)= \\
f_{j_{i}, j_{i}^{\prime}, 1 \leq i \leq \frac{1}{2} n(n+3)-2}^{l_{i}, l_{i}, 1 \leq i \leq n+1 ; l_{i j} 1 \leq i<j \leq n+1}\left(A_{1}, \ldots, A_{N} ; s_{i j}, 1 \leq i<j \leq n+1\right) \times \\
C\left(l_{1}, \ldots, l_{n+1} ; l_{i j}, 1 \leq i<j \leq n+1 ; j_{1}, \ldots, j_{\frac{1}{2} n(n+3)-2} ;\right. \\
\left.m_{1}, \ldots, m_{n+1} ; m_{i j}, 1 \leq i<j \leq n+1\right) \times \\
C\left(\dot{l}_{1}, \ldots, \dot{l}_{n+1} ; l_{i j}, 1 \leq i<j \leq n+1 ; j_{1}^{\prime}, \ldots, j_{\frac{1}{2} n(n+3)-2}^{\prime} ;\right. \\
\left.\dot{m}_{1}, \ldots, \dot{m}_{n+1} ; \dot{m}_{i j}, 1 \leq i<j \leq n+1\right)
\end{array}
$$

where a continuous function

$$
f_{j_{i}, j_{i}^{\prime}, 1 \leq i \leq \frac{1}{2} n(n+3)-2}^{l_{i} i_{i}, 1 \leq i \leq n+1 ; l_{i j} 1 \leq i<j \leq n+1}\left(A_{1}, \ldots, A_{N} ; s_{i j}, 1 \leq i<j \leq n+1\right)
$$

with a support in the product of the positive semi - axes is polynomial bounded; the generalized Clebsch - Gordan coefficient

$$
C\left(l_{1}, \ldots, l_{n_{1}+2} ; l_{n_{1}+3}, \ldots, l_{n_{1}+n_{2}+4} ; j_{1}, \ldots, j_{n_{1}+n_{2}+1} ; m_{1}, \ldots, m_{n_{1}+2} ; m_{n_{1}+3}, \ldots, m_{n_{1}+n_{2}+4}\right)
$$

is given by the relation (2.32).

The relations (2.12), (2.13) imply that the distribution (3.9) for $n=1$ with the functions (3.43) satisfies the covariance relation (2.14). The relations (2.33), (2.34) imply that the distribution (3.9) with the functions (3.44) satisfies the covariance relation (2.14).

\section{Asymptotic condition}

It is proved in the book [2] that Gårding - Wightman axioms [1] including the uniqueness of the vacuum imply

$$
\begin{array}{r}
\lim _{t \rightarrow \infty}\left(\Psi_{0}, \psi_{\mu_{1}}\left(x_{1}\right) \cdots \psi_{\mu_{j}}\left(x_{j}\right) \psi_{\mu_{j+1}}\left(x_{j+1}+t a\right) \psi_{\mu_{j+2}}\left(x_{j+2}+t a\right) \cdots \psi_{\mu_{n}}\left(x_{n}+t a\right) \Psi_{0}\right)= \\
\left(\Psi_{0}, \psi_{\mu_{1}}\left(x_{1}\right) \cdots \psi_{\mu_{j}}\left(x_{j}\right) \Psi_{0}\right)\left(\Psi_{0}, \psi_{\mu_{j+1}}\left(x_{j+1}\right) \psi_{\mu_{j+2}}\left(x_{j+2}\right) \cdots \psi_{\mu_{n}}\left(x_{n}\right) \Psi_{0}\right)
\end{array}
$$

for $a \in \mathbf{R}^{4},(a, a)<0$. The limit (4.1) is convergent in the topology of the space $S^{\prime}\left(\mathbf{R}^{4 n}\right)$.

Let us consider the asymptotic behavior of the vacuum expectation values (3.8), (3.9). Due to ([11], Section 3.1) we define the quasi - asymptotic value of a distribution. Let a distribution $f(x) \in S^{\prime}(\mathbf{R})$ have a support in the positive semi - axis. A distribution $f(x)$ has a quasi - asymptotic value with respect to the transformations $x \rightarrow t^{-1} x$ and the function $\rho(t)=t^{-\lambda}, \lambda$ is a complex number, if in the topology of the space $S^{\prime}(\mathbf{R})$

$$
\lim _{t \rightarrow \infty} t^{\lambda} f\left(t^{-1} x\right)=g(x) .
$$


The limit (4.2) is the characteristic of the asymptotic behavior of the distribution $f(x)$ at zero point. If we choose the transformations $x \rightarrow t x$, then such limit will be the characteristic of the asymptotic behavior of the distribution $f(x)$ at infinity. We can change the function $\rho(t)=t^{-\lambda}$ in the definition (4.2) by any regular varying function ([11], Section 3.2).

The definition (4.2) implies that the distribution $g(x)$ is homogeneous of the degree $\lambda$

$$
g(t x)=t^{\lambda} g(x), t>0 .
$$

For $\operatorname{Re} \lambda>-1$ any homogeneous distribution $g(x) \in S^{\prime}(\mathbf{R})$ of the degree $\lambda$ with a support in the positive semi - axis has the form ([12], Chapter I, Section 3.11)

$$
g(x)=C \frac{\theta(x) x^{\lambda}}{\Gamma(\lambda+1)}
$$

where $C$ is a constant, $\theta(x)$ is the step function (2.17) and $\Gamma(\lambda+1)$ is the gamma - function. Due to ([12], Chapter I, Sections 3.2, 3.5) the function (4.4) of the variable $\lambda$ has the analytic continuation to an entire function. The formula (4.4) is valid for any complex $\lambda$. Due to ([12], Chapter I, Section 3.5)

$$
\left.\frac{\theta(x) x^{\lambda}}{\Gamma(\lambda+1)}\right|_{\lambda=-n}=\delta^{(n-1)}(x), n=1,2, \ldots .
$$

The vacuum expectation values (3.8), (3.9) are defined by the continuous functions (3.10). The quasi - asymptotic value of the continuous function (3.10) we define as the limit of the type (4.2) for the space of the continuous test functions $\phi(x)$ rapidly decreasing: the norm

$$
\sup _{x \in \mathbf{R}}\left(1+x^{2}\right)^{m}|\phi(x)|
$$

is finite for any natural number $m$.

Let an infinitely differentiable function $\phi\left(x_{1}, \ldots, x_{n+1}\right)$ have a compact support in the domain $\left(x_{j}-x_{i}, x_{j}-x_{i}\right)<0,1 \leq i<j \leq n+1$. The relations (3.8), (3.9), (3.15), (3.25) imply

$$
\begin{array}{r}
\int d^{4(n+1)} x W_{m_{1}, \ldots, m_{n+1} ; m_{1}, \ldots, \dot{m}_{n+1}}^{l_{1}, \ldots, l_{n+1} ; i_{1}, \ldots, i_{n+1}}\left(A_{1}, \ldots, A_{N} ; x_{2}-x_{1}, \ldots, x_{n+1}-x_{n}\right) \phi\left(x_{1}, \ldots, x_{n+1}\right)= \\
\sum_{l_{i j} \in 1 / 2 \mathbf{Z}_{+}, 1 \leq i<j \leq n+1} \sum_{m_{i j}, \dot{m}_{i j}=-l_{i j},-l_{i j}+1, \ldots, l_{i j}-1, l_{i j}, 1 \leq i<j \leq n+1} \int d^{4(n+1)} x \phi\left(x_{1}, \ldots, x_{n+1}\right) \times \\
\left(\prod_{1 \leq i<j \leq n+1}\left(2 \pi\left(-\left(x_{j}-x_{i}, x_{j}-x_{i}\right)\right)^{q} t_{m_{i j} \dot{m}_{i j}}^{l_{i j}}\left(\sqrt{-1}\left(\tilde{x}_{i}-\tilde{x}_{j}\right)\right)\right)\right) \times \\
\left(\prod_{1 \leq i<j \leq n+1} \int_{0}^{\infty} \mu_{i j} d \mu_{i j}\right) \\
\int_{m_{i}, \dot{m}_{i}, 1 \leq i \leq n+1 ; m_{i j}, \dot{m}_{i j}, 1 \leq i<j \leq n+1}\left(A_{1}, \ldots, A_{N} ; \mu_{i j}, 1 \leq i<j \leq n+1\right) \times \\
\prod_{1 \leq i<j \leq n+1}\left(-\mu_{i j}\left(x_{j}-x_{i}, x_{j}-x_{i}\right)\right)^{-1 / 2} K_{1}\left(\left(-\mu_{i j}\left(x_{j}-x_{i}, x_{j}-x_{i}\right)\right)^{1 / 2}\right) .
\end{array}
$$

\section{Asymptotic condition}

The vacuum expectation value of the product of $n+1, n=1,2, \ldots$, quantum fields is given by the distribution (3.8), (3.9). 
For any natural numbers $n>n_{1}$, any permutation $\pi$ of the natural numbers $1, \ldots, n+1$ and any continuous rapidly decreasing function $\phi\left(\mu_{i j}, 1 \leq i<j \leq n+1\right)$ the function (3.10) satisfies the following condition

$$
\begin{aligned}
& \lim _{t \rightarrow \infty}\left(\prod_{1 \leq i<j \leq n_{1}+1, n_{1}+1<i<j \leq n+1} \int_{0}^{\infty} d \mu_{\pi(i) \pi(j)}\right) \times \\
& \left(\prod_{1 \leq i \leq n_{1}+1, n_{1}+1<j \leq n+1} \int_{0}^{\infty} t^{q-2+l_{\pi(i) \pi(j)}} d \mu_{\pi(i) \pi(j)}\right) f_{m_{i}, \dot{m}_{i}, 1 \leq i \leq n+1 ; m_{i j}, \dot{m}_{i j}, 1 \leq i<j \leq n+1}^{l_{i} i_{i}, 1 \leq i \leq n+1 ; l_{i j} 1 \leq i<j \leq n+1} \\
& \left(A_{1}, \ldots, A_{N} ; \mu_{\pi(i) \pi(j)}, 1 \leq i<j \leq n_{1}+1, n_{1}+1<i<j \leq n+1 ;\right. \\
& \left.t^{-1} \mu_{\pi(i) \pi(j)}, 1 \leq i \leq n_{1}+1<j \leq n+1\right) \times \\
& \phi\left(\mu_{i j}, 1 \leq i<j \leq n+1\right)=\left(\prod_{1 \leq i<j \leq n+1} \int_{0}^{\infty} d \mu_{i j}\right) \\
& \operatorname{as}(f)_{m_{i}, \dot{m}_{i}, 1 \leq i \leq n+1 ; m_{i j}, \dot{m}_{i j}, 1 \leq i<j \leq n+1}^{l_{i}, i_{i}, 1 \leq i \leq n+1 ; l_{i j}, 1 \leq i<j \leq n+1}\left(\pi ; A_{1}, \ldots, A_{N} ; \mu_{i j}, 1 \leq i<j \leq n+1\right) \times \\
& \phi\left(\mu_{i j}, 1 \leq i<j \leq n+1\right)
\end{aligned}
$$

where a continuous function

$$
\operatorname{as}(f)_{m_{i}, \dot{m}_{i}, 1 \leq i \leq n+1 ; m_{i j}, \dot{m}_{i j}, 1 \leq i<j \leq n+1}^{l_{i} \dot{l}_{i}, 1 \leq i \leq n+1 ; l_{i j}, 1 \leq i<j \leq n+1}\left(\pi ; A_{1}, \ldots, A_{N} ; \mu_{i j}, 1 \leq i<j \leq n+1\right)
$$

is polynomial bounded.

Lemma 4.1. Let the vacuum expectation value of the product of $n+1, n=1,2, \ldots$, quantum field be the distribution (3.8), (3.9) with the finite series. Let the continuous functions (3.10) satisfy the condition (4.7). Let an infinitely differentiable function $\phi\left(x_{\pi(i)}, 1 \leq i \leq n+1\right)$ have a compact support in the domain $\left(x_{\pi(j)}-x_{\pi(i)}, x_{\pi(j)}-x_{\pi(i)}\right)<0,1 \leq i<j \leq n_{1}+1$, $n_{1}+1<i<j \leq n+1$. Then for any natural numbers $n>n_{1}$, any permutation $\pi$ of the natural numbers $1, \ldots, n+1$ and any vector $a \in \mathbf{R}^{4},(a, a)<0$,

$$
\begin{aligned}
& \lim _{t \rightarrow \infty} \int d^{4(n+1)} x W_{m_{1}, \ldots, m_{n+1} ; \dot{m}_{1}, \ldots, \dot{m}_{n+1}}^{l_{1}, \ldots, l_{n+1} ; i_{1}, \ldots, i_{n+1}}\left(A_{1}, \ldots, A_{N} ; x_{2}-x_{1}, \ldots, x_{n+1}-x_{n}\right) \times \\
& \phi\left(x_{\pi(i)}, 1 \leq i \leq n_{1}+1 ; x_{\pi(i)}-t a, n_{1}+1<i \leq n+1\right)= \\
& \sum_{l_{i j} \in 1 / 2 \mathbf{Z}_{+}, 1 \leq i<j \leq n+1} \sum_{m_{i j}, \dot{m}_{i j}=-l_{i j},-l_{i j}+1, \ldots, l_{i j}-1, l_{i j}, 1 \leq i<j \leq n+1} \\
& \left(\prod_{1 \leq i \leq n_{1}+1, n_{1}+1<j \leq n+1}(-(a, a))^{-l_{\pi(i) \pi(j)}} t_{m_{\pi(i) \pi(j)}}^{l_{\pi(i) \pi(j)}}(-\sqrt{-1} \tilde{a})\right) \times \\
& \left(\prod_{1 \leq i<j \leq n_{1}+1, n_{1}+1<i<j \leq n+1} \int_{0}^{\infty} \mu_{\pi(i) \pi(j)} d \mu_{\pi(i) \pi(j)}\right) \int d^{4(n+1)} x \phi\left(x_{\pi(1)}, \ldots, x_{\pi(n+1)}\right) \times \\
& S_{m_{i}, \dot{m}_{i}, 1 \leq i \leq n+1 ; m_{i j}, \dot{m}_{i j}, 1 \leq i<j \leq n+1}^{l_{i}, i_{i}, 1 \leq i \leq n+1 ; l_{i j}, 1 \leq i<j \leq n+1} \\
& \left(\pi ; A_{1}, \ldots, A_{N} ; \mu_{\pi(i) \pi(j)}, 1 \leq i<j \leq n_{1}+1, n_{1}+1<i<j \leq n+1\right) \times \\
& \prod_{1 \leq i<j \leq n_{1}+1, n_{1}+1<i<j \leq n+1}\left(2 \pi\left(-\left(x_{\pi(j)}-x_{\pi(i)}, x_{\pi(j)}-x_{\pi(i)}\right)\right)^{q}\right) \times \\
& t_{m_{\pi(i) \pi(j)}^{l_{\pi(i) \pi(j)}} \dot{m}_{\pi(i) \pi(j)}}\left(\sqrt{-1}\left(\tilde{x}_{\pi(i)}-\tilde{x}_{\pi(j)}\right)\right)\left(-\mu_{\pi(i) \pi(j)}\left(x_{\pi(j)}-x_{\pi(i)}, x_{\pi(j)}-x_{\pi(i)}\right)\right)^{-1 / 2} \times \\
& K_{1}\left(\left(-\mu_{\pi(i) \pi(j)}\left(x_{\pi(j)}-x_{\pi(i)}, x_{\pi(j)}-x_{\pi(i)}\right)\right)^{1 / 2}\right)
\end{aligned}
$$


where the function

$$
\begin{aligned}
& S^{l_{i}, i_{i}, 1 \leq i \leq n+1 ; l_{i j}, 1 \leq i<j \leq n+1} \\
& S_{m_{i}, \dot{m}_{i}}, 1 \leq i \leq n+1 ; m_{i j}, \dot{m}_{i j}, 1 \leq i<j \leq n+1 \\
& \left(\pi ; A_{1}, \ldots, A_{N} ; \mu_{\pi(i) \pi(j)}, 1 \leq i<j \leq n_{1}+1, n_{1}+1<i<j \leq n+1\right)= \\
& \left(\prod_{1 \leq i \leq n_{1}+1, n_{1}+1<j \leq n+1} \int_{0}^{\infty} d \mu_{\pi(i) \pi(j)}\right) \\
& \operatorname{as}(f)_{m_{i}, \dot{m}_{i}, 1 \leq i \leq n+1 ; m_{i j}, \dot{m}_{i j}, 1 \leq i<j \leq n+1}^{l_{i}, i_{i} \leq i \leq n+1 ; l_{i j}, 1 \leq i<j \leq n+1}\left(\pi ; A_{1}, \ldots, A_{N} ; \mu_{i j}, 1 \leq i<j \leq n+1\right) \times \\
& \prod_{1 \leq i \leq n_{1}+1, n_{1}+1<j \leq n+1} 2 \pi\left(\mu_{\pi(i) \pi(j)}\right)^{1 / 2} K_{1}\left(\left(\mu_{\pi(i) \pi(j)}\right)^{1 / 2}\right) .
\end{aligned}
$$

We use the same symbol $\pi$ for the number and a permutation.

Proof. Let an infinitely differentiable function $\phi\left(x_{\pi(i)}, 1 \leq i \leq n+1\right)$ have a compact support in the domain $\left(x_{\pi(j)}-x_{\pi(i)}, x_{\pi(j)}-x_{\pi(i)}\right)<0,1 \leq i<j \leq n_{1}+1, n_{1}+1<i<j \leq n+1$. Then in view of the relation (4.6) for sufficiently large number $t$ and for any vector $a \in \mathbf{R}^{4}$, $(a, a)<0$, we have

$$
\begin{aligned}
& \int d^{4(n+1)} x W_{m_{1}, \ldots, m_{n+1} ; \dot{m}_{1}, \ldots, \dot{m}_{n+1}}^{l_{1}, \ldots, l_{n+1} ; \dot{l}_{1}, \ldots, i_{n+1}}\left(A_{1}, \ldots, A_{N} ; x_{2}-x_{1}, \ldots, x_{n+1}-x_{n}\right) \times \\
& \phi\left(x_{\pi(i)}, 1 \leq i \leq n_{1}+1 ; x_{\pi(i)}-t a, n_{1}+1<i \leq n+1\right)= \\
& \sum_{l_{i j} \in 1 / 2 \mathbf{Z}_{+}, 1 \leq i<j \leq n+1} \sum_{m_{i j}, \dot{m}_{i j}=-l_{i j},-l_{i j}+1, \ldots, l_{i j}-1, l_{i j}, 1 \leq i<j \leq n+1} \\
& \left(\prod_{1 \leq i<j \leq n+1} \int_{0}^{\infty} \mu_{i j} d \mu_{i j}\right) \\
& f_{m_{i}, \dot{m}_{i}, 1 \leq i \leq n+1 ; m_{i j}, m_{i j}, 1 \leq i<j \leq n+1}^{l_{i}, i_{i}, 1 \leq i \leq n+1 ; l_{i j} 1 \leq i<j \leq n+1}\left(A_{1}, \ldots, A_{N} ; \mu_{i j}, 1 \leq i<j \leq n+1\right) \times \\
& \int d^{4(n+1)} x \phi\left(x_{\pi(1)}, \ldots, x_{\pi(n+1)}\right) G(a, \mu, x) H(\mu, x), \\
& G(a, \mu, x)=\prod_{1 \leq i \leq n_{1}+1, n_{1}+1<j \leq n+1}\left(2 \pi\left(-\left(x_{\pi(j)}-x_{\pi(i)}+t a, x_{\pi(j)}-x_{\pi(i)}+t a\right)\right)^{q}\right) \times \\
& t_{m_{\pi(i) \pi(j)} \dot{m}_{\pi(i) \pi(j)}}^{l_{\pi(i) \pi(j)}}\left(\sqrt{-1}\left(\tilde{x}_{\pi(i)}-\tilde{x}_{\pi(j)}-t \tilde{a}\right)\right) \times \\
& \left(-\mu_{\pi(i) \pi(j)}\left(x_{\pi(j)}-x_{\pi(i)}+t a, x_{\pi(j)}-x_{\pi(i)}+t a\right)\right)^{-1 / 2} \times \\
& K_{1}\left(\left(-\mu_{\pi(i) \pi(j)}\left(x_{\pi(j)}-x_{\pi(i)}+t a, x_{\pi(j)}-x_{\pi(i)}+t a\right)\right)^{1 / 2}\right) \text {, } \\
& H(\mu, x)=\prod_{1 \leq i<j \leq n_{1}+1, n_{1}+1<i<j \leq n+1}\left(2 \pi\left(-\left(x_{\pi(j)}-x_{\pi(i)}, x_{\pi(j)}-x_{\pi(i)}\right)\right)^{q}\right) \times
\end{aligned}
$$

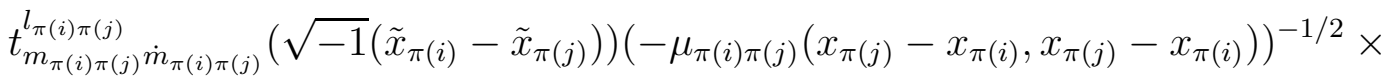

$$
\begin{aligned}
& K_{1}\left(\left(-\mu_{\pi(i) \pi(j)}\left(x_{\pi(j)}-x_{\pi(i)}, x_{\pi(j)}-x_{\pi(i)}\right)\right)^{1 / 2}\right) .
\end{aligned}
$$

The series in the right - hand side of the equality (4.11) are finite. If a vector $x \in \mathbf{R}^{4}$ lies in a compact set in the domain $(x, x)<0$, then in view of the relation (3.23) the function

$$
\mu(-\mu(x, x))^{-1 / 2} K_{1}\left((-\mu(x, x))^{1 / 2}\right)
$$

of the variable $\mu$ rapidly decreases on the positive semi - axis. We change the number $t$ by the number $t(-(a, a))^{-1 / 2}$. Now the relations (4.7), (4.11) imply the relation (4.9). The lemma is proved. 
Let the function (4.10) be not zero only for the spins $l_{\pi(i) \pi(j)}=0,1 \leq i \leq n_{1}+1$, $n_{1}+1<j \leq n+1$ and for these spins

$$
\begin{array}{r}
S_{m_{i}, \dot{m}_{i}, 1 \leq i \leq n+1 ; m_{i j}, \dot{m}_{i j}, 1 \leq i<j \leq n+1}^{l_{i}, \dot{l}_{i}, 1 \leq i \leq n+1 ; l_{i j} 1 \leq i<j \leq n+1} \\
\left(\pi ; A_{1}, \ldots, A_{N} ; \mu_{\pi(i) \pi(j)}, 1 \leq i<j \leq n_{1}+1, n_{1}+1<i<j \leq n+1\right)= \\
f_{m_{\pi(i)}, \dot{m}_{\pi(i)}, 1 \leq i \leq n_{1}+1 ; m_{\pi(i) \pi(j)}, \dot{m}_{\pi(i) \pi(j)}, 1 \leq i<j \leq n_{1}+1}^{l_{\pi(i)}, i_{\pi(i)}, 1 \leq i \leq n_{1}+1 ; l_{\pi(i) \pi(j)} 1 \leq i<j \leq n_{1}+1} \\
\left(\pi\left(A_{1}^{(1)}\right), \ldots, \pi\left(A_{N_{1}}^{(1)}\right) ; \mu_{\pi(i) \pi(j)}, 1 \leq i<j \leq n_{1}+1\right) \times \\
f_{m_{\pi(i)}, \dot{m}_{\pi(i)}, n_{1}+1 \leq i \leq n+1 ; m_{\pi(i) \pi(j)}, \dot{m}_{\pi(i) \pi(j)}, n_{1}+1 \leq i<j \leq n+1}^{l_{\pi(i)}, i_{\pi(i)}, n_{1}+1 \leq i \leq n+1 ; l_{\pi(i) \pi(j)} n_{1}+1 \leq i<j \leq n+1} \\
\left(\pi\left(A_{1}^{(2)}\right), \ldots, \pi\left(A_{N_{2}}^{(2)}\right) ; \mu_{\pi(i) \pi(j)}, n_{1}+1<i<j \leq n+1\right)
\end{array}
$$

The substitution of the function (4.12) into the relation (4.9) yields the relation of the type (4.1).

\section{References}

[1] Wightman, A.S., Gårding, L.: Fields as Operator Valued Distributions in Relativistic Quantum Field Theory. Arkiv f. Fys. 28, 129 - 189 (1964)

[2] Streater, R.F., Wightman, A.S.: PCT, Spin and Statistics and All That. New York: Benjamin, 1964.

[3] Methée, P.D.: Sur les distributions invariantes dans le groupe des rotations de Lorentz. Comment. Math. Helv. 28, 225 - 269 (1954)

[4] Gårding, L., Lions, J.L.: Functional Analysis. Suppl. Nuovo Cim. 14, 9 - 66 (1959)

[5] Zinoviev, Yu.M.: On Lorentz Invariant Distributions. Commun. Math. Phys. 47, 33 $42(1976)$

[6] Zinoviev Yu.M.: Lorentz Covariant Distributions with the Spectral Conditions. hep th/0701153

[7] Vilenkin, N.Ya.: Special Functions and the Theory of Group Representations. Providence, R.I.: American Math. Soc., 1968.

[8] Bros, J., Epstein, H., Glaser, V.: On the Connection Between Analyticity and Lorentz Covariance of Wightman Functions. Commun. Math. Phys. 6, 77 - 100 (1967)

[9] Vladimirov, V.S.: Methods of Theory of Many Complex Variables. Cambridge, MA: MIT Press, 1966.

[10] Bateman, H., Erdélyi, A.S.: Higher Transcendental Functions. Vol. 2, New York Toronto - London: McGraw - Hill, 1953.

[11] Vladimirov V.S., Drozhzhinov J.N., Zavialov B.I.: Tauberian Theorems for Generalized Functions. Dordrecht - Boston - London: Kluwer Academic Publishers, 1988.

[12] Gel'fand, I.M., Shilov, G.E.: Generalized Functions. Vol. 1, Operations on Them. New York: Academic Press, 1964. 